\title{
ON SEMILINEAR CAUCHY PROBLEMS WITH NON-DENSE DOMAIN
}

\author{
Pierre Magal \\ UMR CNRS 5251, IMB \& INRIA sud-ouest Anubis \\ University of Bordeaux, 146 rue Léo Saignat \\ 33076 Bordeaux, France \\ Shigui Ruan \\ Department of Mathematics, University of Miami \\ Coral Gables, FL 33124-4250, USA
}

(Submitted by: Giuseppe Da Prato)

\begin{abstract}
We are interested in studying semilinear Cauchy problems in which the closed linear operator is not Hille-Yosida and its domain is not densely defined. Using integrated semigroup theory, we study the positivity of solutions to the semilinear problem, the Lipschitz perturbation of the problem, differentiability of the solutions with respect to the state variable, time differentiability of the solutions, and the stability of equilibria. The obtained results can be used to study several types of differential equations, including delay differential equations, age-structure models in population dynamics, and evolution equations with nonlinear boundary conditions.
\end{abstract}

\section{INTRODUCTION}

The main purpose of this paper is to present a comprehensive semilinear theory (Cazenave and Haraux [5], Davies and Pang [8], Hieber [15, 16], Xiao and Liang [45], etc.) that will allow us to study the dynamics of nondensely defined Cauchy problems, such as asymptotic behavior of solutions and bifurcations. Consider the Cauchy problem:

$$
\frac{d u}{d t}=A u+F(t, u), \quad t \geq 0, u(0)=u_{0} \in \overline{D(A)},
$$

Accepted for publication: December 2008.

AMS Subject Classifications: 35K57, 45D05, 47D06.

Research was partially supported by NSF grant DMS-0715772. 
where $A: D(A) \subset X \rightarrow X$ is a linear operator in a Banach space $X$ and $F:[0,+\infty) \times \overline{D(A)} \rightarrow X$ is a continuous map. We are interested in studying the problem when $\overline{D(A)}$ is not dense in $X$ and $A$ is not a Hille-Yosida operator. Several types of differential equations, such as delay differential equations, age-structure models in population dynamics, some partial differential equations, evolution equations with nonlinear boundary conditions, can be written as semilinear Cauchy problems with non-dense domain (see Da Prato and Sinestrari [7], Thieme [35, 36], Liu et al. [19], Magal and Ruan [22]).

When $A$ is a Hille-Yosida operator (i.e., if there exist two constants $\omega \in \mathbb{R}$ and $M \geq 1$ such that $(\omega,+\infty) \subset \rho(A)$ and $\left\|(\lambda I-A)^{-k}\right\|_{\mathcal{L}(X)} \leq \frac{M}{(\lambda-\omega)^{k}}$ for all $\lambda>\omega, k \geq 1$, where $\mathcal{L}(X)$ is the space of bounded linear operators from $X$ into $X$ and $\rho(A)$ is the resolvent set of $A$ ) and is densely defined (i.e., $\overline{D(A)}=X$ ), the problem has been extensively studied (see Segal [33], Weissler [44], Martin [25], Pazy [30], Hirsch and Smith [17]). When $A$ is a Hille-Yosida operator but its domain is non-densely defined, Da Prato and Sinestrari [7] investigated the existence of several types of solutions for (1.1). Thieme [35] investigated the semilinear Cauchy problem with a Lipschitz perturbation of the closed linear operator $A$ which is non-densely defined but is Hille-Yosida. Integrated semigroup theory was used to obtain a variation of constants formula which allows one to transform the integrated solutions of the evolution equation into solutions of an abstract semilinear Volterra integral equation, which in turn was used to find integrated solutions to the Cauchy problem. Moreover, sufficient and necessary conditions for the invariance of closed convex sets under the solution flow were found. Conditions for the regularity of the solution flow in time and initial state were derived. The steady states of the solution flow were characterized and sufficient conditions for local stability and instability were given. See also Thieme [36, 38].

In this paper, we attempt to extend Thieme's results [35] to the case when the operator $A$ is not Hille-Yosida. The perturbations of the operator are only Lipschitz on bounded sets. In order to do such extensions, we need to obtain an estimate for nonhomogeneous equations which is obtained in Proposition 2.14. Then we are able to develop and extend most of his results (except the positivity result) in [35]. We obtain some weak and classical conditions for positivity of the solutions by considering the problem in a more general setting; that is, we only impose the condition that the integrated solutions exist when the nonlinear interaction term $F$ is continuous. 
We would like to make some comments about the assumption that the linear operator $A$ is not a Hille-Yosida operator. We first assume that the resolvent set $\rho(A)$ of $A$ is non-empty and that $A_{0}$, the part of $A$ in $\overline{D(A)}$, is the infinitesimal generator of a strongly continuous semigroup $\left\{T_{A_{0}}(t)\right\}_{t \geq 0}$ of bounded linear operators on $\overline{D(A)}$. Then $A$ generates an integrated semigroup $\left\{S_{A}(t)\right\}_{t \geq 0}$ on $X$, defined by

$$
S_{A}(t)=\left(\lambda I-A_{0}\right) \int_{0}^{t} T_{A_{0}}(l) d l(\lambda I-A)^{-1}
$$

for each $\lambda \in \rho(A)$.

Then (see Magal and Ruan [22] and Thieme [38]) we need to impose an additional condition to assure the existence of integrated solutions of the non-homogeneous Cauchy problem

$$
\frac{d u(t)}{d t}=A u(t)+f(t) \text { for } t \geq 0 \text { and } u(0)=0 .
$$

Here we assume that $A$ is not a Hille-Yosida operator (Assumption 2.1), but for each $f \in C([0, \tau], X)$ the Cauchy problem (1.2) has an integrated solution $u_{f}(t)$, and there exists a map $\delta:[0,+\infty) \rightarrow[0,+\infty)$ (independent of $f$ ) such that (Assumption 2.8 and Theorem 2.9)

$$
\left\|u_{f}(t)\right\| \leq \delta(t) \sup _{s \in[0, t]}\|f(s)\|
$$

where $\delta(t) \rightarrow 0$ as $t \rightarrow 0$. In particular as in the example presented in Magal and Ruan [22], the Cauchy problem may not have an integrated solution for $f \in L^{1}((0, \tau) ; X)$. The goal of this paper is to show that under such a condition on the Cauchy problem (1.2), we still can extend the results for the classical semi-linear Cauchy problems.

In practice, it is relatively easy to verify that the linear operator $A$ is not a Hille-Yosida operator (i.e., satisfying Assumption 2.1). Nevertheless, for a given example, it may require some work to verify the condition (1.3) (i.e., Assumption 2.8). In the context of age-structured models the condition (1.3) has been successfully verified by Magal and Ruan [22] using some characterization on the resolvent of $A$. For the same class of PDEs, Thieme [38] also successfully applied the notion of integrated semigroup with bounded $p$ semi-variation to verify condition (1.3). For parabolic systems, this question has been studied by Prevost [32] and Ducrot et al. [13]. In particular, it has been proved that if $A$ is almost sectorial and $A_{0}$, the part of $A$ in $D(A)$, is a sectorial operator, then condition (1.3) is satisfied. We also refer to Prevost [32] for more examples in the context of parabolic equations. 
The rest of the paper is organized as follows. In section 2, we recall some results on integrated semigroups and give an estimate for solutions to the nonhomogeneous equation (see Proposition 2.14) which is crucial for the stability of equilibria to the semilinear problem. Sections 3-7 are devoted to the study of the semilinear problem. In section 3, positivity of solutions to the semilinear problem is considered. Section 4 focuses on Lipschitz perturbations of the problem. Section 5 deals with differentiability of the solutions with respect to the state variable. In section 6 we are concerned with time differentiability of the solutions. The stability of equilibria is studied in section 7 . In section 8 , as applications we discuss transport equations with nonlinear boundary conditions and parabolic equations with nonlocal boundary conditions and show that our results apply. In particular, we verify that our main Assumptions 2.1 and 2.8 hold for these two types of equations.

Notice that Magal and Ruan [22] presented some techniques and results for integrated semigroups when the generator is not a Hille-Yosida operator and is non-densely defined, obtained necessary and sufficient conditions for the existence of mild solutions for non-densely defined non-homogeneous Cauchy problems, and applied the results to study age structured models. Recently, Magal and Ruan [23] developed the center manifold theory for non-densely defined Cauchy problems and employed the theory to establish a Hopf bifurcation theorem for age structured models. This paper complements our previous articles $[22,23]$ in studying semilinear Cauchy problems with non-dense domain.

\section{Integrated Semigroups}

In this section we recall some results about integrated semigroups. We refer to Arendt [2, 3], Neubrander [28], Kellermann and Hieber [18], Thieme $[36,38]$, Arendt et al. [4], and Magal and Ruan [22, 23] for more detailed results on the subject.

Let $X$ and $Z$ be two Banach spaces. Let $\mathcal{L}(X, Z)$ denote the space of bounded linear operators from $X$ into $Z$ and by $\mathcal{L}(X)$ the space $\mathcal{L}(X, X)$. Let $A: D(A) \subset X \rightarrow X$ be a linear operator. If $A$ is the infinitesimal generator of a strongly continuous semigroup of bounded linear operators on $X$, let $\left\{T_{A}(t)\right\}_{t \geq 0}$ denote this semigroup. The resolvent set of $A$ is denoted by $\rho(A)=\{\lambda \in \mathbb{C}: \lambda I-A$ is invertible $\}$. Set $X_{0}:=\overline{D(A)}$ and $A_{0}$ the part of $A$ in $X_{0}$, which is a linear operator on $X_{0}$ defined by

$$
A_{0} x=A x, \forall x \in D\left(A_{0}\right):=\left\{y \in D(A): A y \in X_{0}\right\} .
$$


Assume that $(\omega,+\infty) \subset \rho(A)$. Then it is easy to check that, for each $\lambda>\omega$,

$$
D\left(A_{0}\right)=(\lambda I-A)^{-1} X_{0} \text { and }\left(\lambda I-A_{0}\right)^{-1}=\left.(\lambda I-A)^{-1}\right|_{X_{0}} .
$$

Recall that $A$ is a Hille-Yosida operator if there exist two constants, $\omega \in \mathbb{R}$ and $M \geq 1$, such that $(\omega,+\infty) \subset \rho(A)$ and

$$
\left\|(\lambda I-A)^{-k}\right\|_{\mathcal{L}(X)} \leq \frac{M}{(\lambda-\omega)^{k}}, \quad \forall \lambda>\omega, \quad \forall k \geq 1 .
$$

In the following, we assume that $A$ satisfies some weaker conditions.

Assumption 2.1. Assume that $A: D(A) \subset X \rightarrow X$ is a linear operator on a Banach space $(X,\|\|$.$) satisfying the following properties:$

(a) There exist two constants, $\omega_{A} \in \mathbb{R}$ and $M_{A} \geq 1$, such that $\left(\omega_{A},+\infty\right)$ $\subset \rho(A)$ and

$$
\left\|(\lambda I-A)^{-k}\right\|_{\mathcal{L}\left(X_{0}\right)} \leq \frac{M_{A}}{\left(\lambda-\omega_{A}\right)^{k}}, \quad \forall \lambda>\omega_{A}, \quad \forall k \geq 1 ;
$$

(b) $\lim _{\lambda \rightarrow+\infty}(\lambda I-A)^{-1} x=0, \forall x \in X$.

By using Lemma 2.1 in Magal and Ruan [22] and Assumption 2.1-(b) we deduce that $\overline{D\left(A_{0}\right)}=X_{0}$. By the Hille-Yosida theorem (see Pazy [30], Theorem 5.3 on page 20) and the fact that if $\rho(A) \neq \emptyset$ then $\rho(A)=\rho\left(A_{0}\right)$ (see Magal and Ruan [23, Lemma 2.4]), one obtains the following lemma.

Lemma 2.2. Assumption 2.1 is satisfied if and only if $\rho(A) \neq \emptyset, A_{0}$ is the infinitesimal generator of a linear $C_{0}$-semigroup $\left\{T_{A_{0}}(t)\right\}_{t \geq 0}$ on $X_{0}$, and

$$
\left\|T_{A_{0}}(t)\right\| \leq M_{A} e^{\omega_{A} t}, \forall t \geq 0 .
$$

Now we give the definition of an integrated semigroup.

Definition 2.3. Let $(X,\|\|$.$) be a Banach space. A family of bounded linear$ operators $\{S(t)\}_{t \geq 0}$ on $X$ is called an integrated semigroup if the following hold.

(i) $S(0)=0$.

(ii) The map $t \rightarrow S(t) x$ is continuous on $[0,+\infty)$ for each $x \in X$.

(iii) $S(t)$ satisfies

$$
S(s) S(t)=\int_{0}^{s}(S(r+t)-S(r)) d r, \quad \forall t, s \geq 0
$$


An integrated semigroup $\{S(t)\}_{t>0}$ is said to be non-degenerate if $S(t) x=$ 0 for all $t \geq 0$, then $x=0$. According to Thieme [36], a linear operator $A$ : $D(A) \subset X \rightarrow X$ is the generator of a non-degenerate integrated semigroup $\{S(t)\}_{t \geq 0}$ on $X$ if and only if

$$
x \in D(A), y=A x \Leftrightarrow S(t) x-t x=\int_{0}^{t} S(s) y d s, \forall t \geq 0 .
$$

From [36, Lemma 2.5], we know that, if $A$ generates $\left\{S_{A}(t)\right\}_{t \geq 0}$, then for each $x \in X$ and $t \geq 0$,

$$
\int_{0}^{t} S_{A}(s) x d s \in D(A) \text { and } S(t) x=A \int_{0}^{t} S_{A}(s) x d s+t x .
$$

An integrated semigroup $\{S(t)\}_{t>0}$ is said to be exponentially bounded if there exist two constants, $\widehat{M}>0$ and $\widehat{\omega}>0$, such that

$$
\|S(t)\|_{\mathcal{L}(X)} \leq \widehat{M} e^{\widehat{\omega} t}, \quad \forall t \geq 0
$$

When we restrict ourselves to the class of non-degenerate exponentially bounded integrated semigroups, Thieme's notion of generator is equivalent to the one introduced by Arendt [3]. More precisely, combining Theorem 3.1 in Arendt [3] and Proposition 3.10 in Thieme [36], one has the following result.

Theorem 2.4. Let $\{S(t)\}_{t \geq 0}$ be a strongly continuous exponentially bounded family of bounded linear operators on a Banach space $(X,\|\|$.$) and A$ : $D(A) \subset X \rightarrow X$ be a linear operator. Then $\{S(t)\}_{t \geq 0}$ is a non-degenerate integrated semigroup and $A$ its generator if and only if there exists some $\widehat{\omega}>0$ such that $(\widehat{\omega},+\infty) \subset \rho(A)$ and

$$
(\lambda I-A)^{-1} x=\lambda \int_{0}^{\infty} e^{-\lambda s} S(s) x d s, \quad \forall \lambda>\hat{\omega} .
$$

The following result is well known in the context of integrated semigroups.

Proposition 2.5. Let Assumption 2.1 be satisfied. Then A generates a uniquely determined non-degenerate exponentially bounded integrated semigroup $\left\{S_{A}(t)\right\}_{t \geq 0}$. Moreover, for each $x \in X$, each $t \geq 0$, and each $\mu>\omega_{A}$, $S_{A}(t) x$ is given by

$$
S_{A}(t) x=\mu \int_{0}^{t} T_{A_{0}}(s)(\mu I-A)^{-1} x d s+\left[I-T_{A_{0}}(t)\right](\mu I-A)^{-1} x .
$$


Furthermore, the map $t \rightarrow S_{A}(t) x$ is continuously differentiable if and only if $x \in X_{0}$ and

$$
\frac{d S_{A}(t) x}{d t}=T_{A_{0}}(t) x, \quad \forall t \geq 0, \forall x \in X_{0} .
$$

From now on we define

$$
\left(S_{A} * f\right)(t)=\int_{0}^{t} S_{A}(t-s) f(s) d s, \forall t \in[0, \tau],
$$

whenever $f \in L^{1}((0, \tau), X)$.

We now consider the non-homogeneous Cauchy problem

$$
\frac{d u}{d t}=A u(t)+f(t), t \in[0, \tau], u(0)=x \in \overline{D(A)}
$$

and assume that $f$ belongs to some appropriate subspace of $L^{1}((0, \tau), X)$.

Definition 2.6. A continuous map $u \in C([0, \tau], X)$ is called an integrated solution (or mild solution) of (2.5) if and only if

$$
\int_{0}^{t} u(s) d s \in D(A), \quad \forall t \in[0, \tau]
$$

and

$$
u(t)=x+A \int_{0}^{t} u(s) d s+\int_{0}^{t} f(s) d s, \forall t \in[0, \tau] .
$$

Since $A$ generates a non-degenerate integrated semigroup on $X$, we can apply Theorem 3.7 in Thieme [36] and obtain the following result.

Lemma 2.7. Let Assumption 2.1 be satisfied. Then for each $x \in \overline{D(A)}$ and each $f \in L^{1}\left(\left(0, \tau_{0}\right), X\right),(2.5)$ has at most one integrated solution.

Denote

$$
\left(S_{A} \diamond f\right)(t)=\frac{d}{d t}\left(S_{A} * f\right)(t)
$$

whenever the map $t \rightarrow\left(S_{A} * f\right)(t)$ is continuously differentiable.

We will say that $\left\{S_{A}(t)\right\}_{t \geq 0}$ has a bounded semi-variation on $[0, t]$ if

$$
V^{\infty}\left(S_{A}, 0, t\right):=\sup \left\{\left\|\sum_{i=1}^{n}\left(S_{A}\left(t_{i}\right)-S_{A}\left(t_{i-1}\right)\right) x_{i}\right\|\right\}<+\infty,
$$

where the supremum is taken over all partitions $0=t_{0}<. .<t_{n}=t$ of the interval $[a, b]$ and over any $\left(x_{1}, . ., x_{n}\right) \in X^{n}$ with $\left\|x_{i}\right\|_{X} \leq 1$, for all $i=1, . ., n$.

In the sequel, we will only assume that (2.5) has an integrated solution whenever $f \in C([0, \tau], X)$, so we will make the following assumption. 
Assumption 2.8. Let $\tau_{0}>0$ be fixed. Assume that $\left\{S_{A}(t)\right\}_{t \geq 0}$ has a bounded semi-variation on $\left[0, \tau_{0}\right]$ and

$$
\lim _{t(>0) \rightarrow 0} V^{\infty}\left(S_{A}, 0, t\right)=0 .
$$

The following theorem, proved by Thieme [38], provides an equivalent condition to Assumption 2.8 and is very helpful in applications. We refer to Magal and Ruan [22], Prevost [32], and Ducrot et al. [13] for verifying Assumption 2.8 for age-structured models and parabolic equations.

Theorem 2.9. Let Assumption 2.1 be satisfied. Then Assumption 2.8 is satisfied if and only if for each $f \in C\left(\left[0, \tau_{0}\right], X\right)$, the map $t \rightarrow\left(S_{A} * f\right)(t)$ is continuously differentiable on $\left[0, \tau_{0}\right]$ and

$$
\left\|\left(S_{A} \diamond f\right)(t)\right\| \leq \delta(t) \sup _{s \in[0, t]}\|f(s)\|, \forall t \in\left[0, \tau_{0}\right]
$$

where $\delta:\left[0, \tau_{0}\right] \rightarrow[0,+\infty)$ is a non-decreasing map satisfying

$$
\lim _{t(>0) \rightarrow 0} \delta(t)=0 .
$$

Proof. Assume first that Assumption 2.8 is satisfied. By using Lemma 3.1 in [38], we have for each $t \in\left[0, \tau_{0}\right]$ and each $f \in C\left(\left[0, \tau_{0}\right], X\right)$ that

$$
\left\|\left(S_{A} \star f\right)(t)\right\| \leq V^{\infty}\left(S_{A}, 0, t\right) \sup _{s \in[0, t]}\|f(s)\|,
$$

where $\left(S_{A} \star f\right)(t)$ is defined as a Stieltjes convolution (see [38]). Moreover, by Theorem 4.2 in [38], we deduce that for each $f \in C\left(\left[0, \tau_{0}\right], X\right)$, the map $t \rightarrow\left(S_{A} * f\right)(t)$ is continuously differentiable and

$$
\left(S_{A} \star f\right)(t)=\left(S_{A} \diamond f\right)(t):=\frac{d}{d t}\left(S_{A} * f\right)(t) .
$$

So by fixing $\delta(t)=V^{\infty}\left(S_{A}, 0, t\right)$ we obtain the desired estimate.

Conversely, by using the same arguments as in the proof of Theorem 3.4 in [38], one deduces that $V^{\infty}\left(S_{A}(), 0, t.\right) \leq \delta(t)$ for all $t \in\left[0, \tau_{0}\right]$, and the result follows.

We have $S_{A}(\tau+h)=S_{A}(\tau)+T_{A_{0}}(\tau) S_{A}(h)$ for all $h \geq 0$, so by using Assumption 2.8 we deduce that $t \rightarrow S_{A}(t)$ has a bounded semi-variation on $\left[0,2 \tau_{0}\right]$. Now, using induction arguments, we deduce that $t \rightarrow S_{A}(t)$ has a bounded semi-variation on $[0, \tau]$ for each $\tau \geq 0$, and by using Theorem 2.9 we obtain the following result. 
Theorem 2.10. Let Assumptions 2.1 and 2.8 be satisfied. Then, for each $\tau>0, t \rightarrow S_{A}(t)$ has a bounded semi-variation on $[0, \tau]$. Moreover, for each $f \in C([0, \tau], X)$, the map $t \rightarrow\left(S_{A} * f\right)(t)$ is continuously differentiable, $\left(S_{A} * f\right)(t) \in D(A)$ for all $t \in[0, \tau]$, and $u(t)=\left(S_{A} \diamond f\right)(t)$ satisfies

$$
u(t)=A \int_{0}^{t} u(s) d s+\int_{0}^{t} f(s) d s, \forall t \in[0, \tau]
$$

and

$$
\|u(t)\| \leq V^{\infty}\left(S_{A}, 0, t\right) \sup _{s \in[0, t]}\|f(s)\|, \forall t \in[0, \tau] .
$$

Furthermore, for each $\lambda \in(\omega,+\infty)$, we have

$$
(\lambda I-A)^{-1}\left(S_{A} \diamond f\right)(t)=\int_{0}^{t} T_{A_{0}}(t-s)(\lambda I-A)^{-1} f(s) d s .
$$

Corollary 2.11. Let Assumptions 2.1 and 2.8 be satisfied. Then, for each $x \in X_{0}$ and each $f \in C\left([0, \tau], X_{0}\right)$, the Cauchy problem (2.5) has a unique integrated solution $u \in C\left([0, \tau], X_{0}\right)$ given by

$$
u(t)=T_{A_{0}}(t) x+\left(S_{A} \diamond f\right)(t), \forall t \in[0, \tau] .
$$

Moreover, we have

$$
\|u(t)\| \leq M_{A} e^{\omega t}\|x\|+V^{\infty}\left(S_{A}, 0, t\right) \sup _{s \in[0, t]}\|f(s)\|, \forall t \in[0, \tau] .
$$

We now consider a bounded perturbation of $A$. The following result was proved in Magal and Ruan [22, Theorem 3.1], which is also closely related to Desch and Schappacher's theorem (see [10] or Engel and Nagel [14, Theorem 3.1 , page 183]).

Theorem 2.12. Let Assumptions 2.1 and 2.8 hold. Let $L \in \mathcal{L}\left(X_{0}, X\right)$. Then $A+L: D(A) \subset X \rightarrow X$ also satisfies Assumptions 2.1 and 2.8 . More precisely, if $\left\{S_{A+L}(t)\right\}_{t \geq 0}$ denotes the integrated semigroup generated by $A+L$, and $\tau_{1} \in\left(0, \tau_{0}\right]$ is chosen such that $\|L\|_{\mathcal{L}\left(X_{0}, X\right)} V^{\infty}\left(S_{A}, 0, \tau_{1}\right)<1$, then, for each $f \in C\left(\left[0, \tau_{1}\right], X\right)$,

$$
\left\|\left(S_{A+L} \diamond f\right)(t)\right\| \leq \frac{V^{\infty}\left(S_{A}, 0, t\right)}{1-\|L\|_{\mathcal{L}\left(X_{0}, X\right)} V^{\infty}\left(S_{A}, 0, \tau_{1}\right)} \sup _{s \in[0, t]}\|f(s)\|, \forall t \in\left[0, \tau_{1}\right] .
$$

We have the following lemma.

Lemma 2.13. Let Assumptions 2.1 and 2.8 be satisfied. Then $t \rightarrow S_{A}(t)$ is continuous from $[0,+\infty)$ into $\mathcal{L}(X)$ and

$$
\lim _{\lambda \rightarrow+\infty}\left\|(\lambda I-A)^{-1}\right\|_{\mathcal{L}(X)}=0 .
$$


Proof. By Proposition 3.10 in Thieme [36], we have for each $\lambda>\max \left(0, \omega_{A}\right)$ that

Note that

$$
(\lambda I-A)^{-1} x=\lambda \int_{0}^{+\infty} e^{-\lambda t} S_{A}(t) x d t
$$

$$
S_{A}(t) x=\frac{d}{d t} \int_{0}^{t} S_{A}(t-s) x d s
$$

so, by Assumption 2.8, we have

$$
\left\|S_{A}(t) x\right\| \leq V^{\infty}\left(S_{A}, 0, t\right)\|x\|, \quad \forall t \geq 0
$$

But

$$
S_{A}(t+h)-S_{A}(t)=T_{A_{0}}(t) S_{A}(h) ;
$$

it follows that $t \rightarrow S_{A}(t)$ is operator norm continuous. Let $\varepsilon>0$ be fixed and let $\tau_{\varepsilon}>0$ be such that $V^{\infty}\left(S_{A}, 0, \tau_{\varepsilon}\right) \leq \varepsilon$. Choose $\gamma>\max \left(0, \omega_{A}\right)$ and $M_{\gamma}>0$ such that

$$
\left\|S_{A}(t) x\right\| \leq M_{\gamma} e^{\gamma t}, \quad \forall t \geq 0 .
$$

Then we have for each $\lambda>\gamma$ that

$$
\left\|(\lambda I-A)^{-1} x\right\| \leq \lambda\left[M_{\gamma} \int_{\tau_{\varepsilon}}^{+\infty} e^{(\gamma-\lambda) t} d t+\varepsilon \int_{0}^{\tau_{\varepsilon}} e^{-\lambda t} d t\right]\|x\| .
$$

Thus,

$$
\limsup _{\lambda \rightarrow+\infty}\left\|(\lambda I-A)^{-1}\right\|_{\mathcal{L}(X)} \leq \varepsilon
$$

This proves the lemma.

By using the fact that $\left(S_{A} \diamond f\right)(t) \in X_{0}$ for all $t \in[0, \tau]$, and formula (2.6), we have for each $f \in C([0, \tau], X)$ that

$$
\left(S_{A} \diamond f\right)(t)=\lim _{\mu \rightarrow+\infty} \int_{0}^{t} T_{A_{0}}(t-l) \mu(\mu I-A)^{-1} f(l) d l, \forall t \in[0, \tau] .
$$

This approximation formula was already observed by Thieme [35] in the classical context of integrated semigroups generated by a Hille-Yosida operator. From this approximation formulation, we then deduce that for each pair $t, s \in[0, \tau]$ with $s \leq t$, and $f \in C([0, \tau], X)$,

$$
\left(S_{A} \diamond f\right)(t)=T_{A_{0}}(t-s)\left(S_{A} \diamond f\right)(s)+\left(S_{A} \diamond f(s+.)\right)(t-s) .
$$

Let $|$.$| denote the norm on X_{0}$ defined by

$$
|x|=\sup _{l \geq 0} e^{-\omega_{A} l}\left\|T_{A_{0}}(l) x\right\|, \quad \forall x \in X_{0} .
$$


Clearly, we have

$$
\left|T_{A_{0}}(t)\right|_{\mathcal{L}\left(X_{0}\right)} \leq e^{\omega_{A} t}, \quad \forall t \geq 0, \quad\|x\| \leq|x| \leq M_{A}\|x\|, \quad \forall x \in X_{0},
$$

and by Assumption 2.8, for each $f \in C([0, \tau], X)$ and each $t \in[0, \tau]$,

$$
\left|\left(S_{A} \diamond f\right)(t)\right| \leq M_{A}\left\|\left(S_{A} \diamond f\right)(t)\right\| \leq M_{A} V^{\infty}\left(S_{A}, 0, t\right) \sup _{s \in[0, t]}\|f(s)\| .
$$

The following proposition is one of the main tools for studying semilinear problems (see the next section and also Magal and Ruan [23] for another class of applications of this result).

Proposition 2.14. Let Assumptions 2.1 and 2.8 be satisfied. Let $\varepsilon>0$ be fixed. Then, for each $\tau_{\varepsilon}>0$ satisfying $M_{A} V^{\infty}\left(S_{A}, 0, \tau_{\varepsilon}\right) \leq \varepsilon$, we have

$$
\left\|\left(S_{A} \diamond f\right)(t)\right\| \leq C(\varepsilon, \gamma) \sup _{s \in[0, t]} e^{\gamma(t-s)}\|f(s)\|, \quad \forall t \geq 0
$$

whenever $\gamma \in\left(\omega_{A},+\infty\right), f \in C\left(\mathbb{R}_{+}, X\right)$, with

$$
C(\varepsilon, \gamma):=\frac{2 \varepsilon \max \left(1, e^{-\gamma \tau_{\varepsilon}}\right)}{1-e^{\left(\omega_{A}-\gamma\right) \tau_{\varepsilon}}} .
$$

Proof. Let $\varepsilon>0, f \in C\left(\mathbb{R}_{+}, X\right)$, and $\gamma>\omega_{A}$ be fixed. Let $\tau_{\varepsilon}=\tau_{\varepsilon}(\varepsilon) \in$ $(0, \tau]$ be such that $M_{A} V^{\infty}\left(S_{A}, 0, \tau_{\varepsilon}\right) \leq \varepsilon$. By $(2.9)$, we have

$$
\left|\left(S_{A} \diamond f\right)(t)\right| \leq \varepsilon \sup _{s \in[0, t]}\|f(s)\|, \forall t \in\left[0, \tau_{\varepsilon}\right] .
$$

Let $\gamma>\omega_{A}$ be fixed. Set $\varepsilon_{1}=\varepsilon \max \left(1, e^{-\gamma \tau_{\varepsilon}}\right)$. Let $k \in \mathbb{N}$ and $t \in\left[k \tau_{\varepsilon},(k+\right.$ 1) $\left.\tau_{\varepsilon}\right]$ be fixed. First, notice that if $\gamma \geq 0$, we have

$$
\begin{aligned}
\varepsilon \sup _{s \in\left[k \tau_{\varepsilon}, t\right]}\|f(s)\| & =\varepsilon \sup _{s \in\left[k \tau_{\varepsilon}, t\right]} e^{\gamma s} e^{-\gamma s}\|f(s)\| \leq \varepsilon e^{\gamma t} \sup _{s \in\left[k \tau_{\varepsilon}, t\right]} e^{-\gamma s}\|f(s)\| \\
& =\varepsilon_{1} e^{\gamma t} \sup _{s \in\left[k \tau_{\varepsilon}, t\right]} e^{-\gamma s}\|f(s)\| .
\end{aligned}
$$

Moreover, if $\gamma<0$, we have

$$
\begin{aligned}
\varepsilon \sup _{s \in\left[k \tau_{\varepsilon}, t\right]}\|f(s)\| & =\varepsilon \sup _{s \in\left[k \tau_{\varepsilon}, t\right]} e^{\gamma s} e^{-\gamma s}\|f(s)\| \leq \varepsilon e^{\gamma k \tau_{\varepsilon}} \sup _{s \in\left[k \tau_{\varepsilon}, t\right]} e^{-\gamma s}\|f(s)\| \\
& =\varepsilon e^{\gamma t} e^{-\gamma t} e^{\gamma k \tau_{\varepsilon}} \sup _{s \in\left[k \tau_{\varepsilon}, t\right]} e^{-\gamma s}\|f(s)\| \\
& =e^{\gamma t} \varepsilon e^{-\gamma\left(t-k \tau_{\varepsilon}\right)} \sup _{s \in\left[k \tau_{\varepsilon}, t\right]} e^{-\gamma s}\|f(s)\| \\
& \leq e^{\gamma t} \varepsilon e^{-\gamma \tau_{\varepsilon}} \sup _{s \in\left[k \tau_{\varepsilon}, t\right]} e^{-\gamma s}\|f(s)\|=e^{\gamma t} \varepsilon_{1} \sup _{s \in\left[k \tau_{\varepsilon}, t\right]} e^{-\gamma s}\|f(s)\| .
\end{aligned}
$$


Therefore, for each $k \in \mathbb{N}$, each $t \in\left[k \tau_{\varepsilon},(k+1) \tau_{\varepsilon}\right]$, and each $\gamma \in \mathbb{R}$, we obtain

$$
\varepsilon \sup _{s \in\left[k \tau_{\varepsilon}, t\right]}\|f(s)\| \leq e^{\gamma t} \varepsilon_{1} \sup _{s \in\left[k \tau_{\varepsilon}, t\right]} e^{-\gamma s}\|f(s)\| .
$$

It follows from (2.10) and (2.11) that, for all $t \in\left[0, \tau_{\varepsilon}\right]$,

$$
\left|\left(S_{A} \diamond f\right)(t)\right| \leq \varepsilon \sup _{s \in[0, t]}\|f(s)\|=e^{\gamma t} \varepsilon_{1} \sup _{s \in[0, t]} e^{-\gamma s}\|f(s)\| .
$$

Using (2.8) with $s=\tau_{\varepsilon}$, we have for all $t \in\left[\tau_{\varepsilon}, 2 \tau_{\varepsilon}\right]$ that

$$
\left(S_{A} \diamond f\right)(t)=T_{0}\left(t-\tau_{\varepsilon}\right)\left(S_{A} \diamond f\right)\left(\tau_{\varepsilon}\right)+\left(S_{A} \diamond f\left(\tau_{\varepsilon}+.\right)\right)\left(t-\tau_{\varepsilon}\right) .
$$

Using (2.10), (2.11), and (2.12), we have

$$
\begin{aligned}
\left|\left(S_{A} \diamond f\right)(t)\right| & \leq e^{\omega_{A}\left(t-\tau_{\varepsilon}\right)}\left|\left(S_{A} \diamond f\right)\left(\tau_{\varepsilon}\right)\right|+\left|\left(S_{A} \diamond f\left(\tau_{\varepsilon}+.\right)\right)\left(t-\tau_{\varepsilon}\right)\right| \\
& \leq e^{\omega_{A}\left(t-\tau_{\varepsilon}\right)} e^{\gamma \tau_{\varepsilon}} \varepsilon_{1} \sup _{s \in\left[0, \tau_{\varepsilon}\right]} e^{-\gamma s}\|f(s)\|+\varepsilon \sup _{s \in\left[\tau_{\varepsilon}, t\right]}\|f(s)\| \\
& \leq e^{\omega_{A}\left(t-\tau_{\varepsilon}\right)} e^{\gamma \tau_{\varepsilon}} \varepsilon_{1} \sup _{s \in\left[0, \tau_{\varepsilon}\right]} e^{-\gamma s}\|f(s)\|+e^{\gamma t} \varepsilon_{1} \sup _{s \in\left[\tau_{\varepsilon}, t\right]} e^{-\gamma s}\|f(s)\| \\
& \leq \varepsilon_{1} e^{\gamma t}\left(e^{\left(\omega_{A}-\gamma\right)\left(t-\tau_{\varepsilon}\right)}+1\right) \sup _{s \in[0, t]} e^{-\gamma s}\|f(s)\| .
\end{aligned}
$$

Similarly, for all $t \in\left[2 \tau_{\varepsilon}, 3 \tau_{\varepsilon}\right]$,

$$
\left(S_{A} \diamond f\right)(t)=T_{A_{0}}\left(t-2 \tau_{\varepsilon}\right)\left(S_{A} \diamond f\right)\left(2 \tau_{\varepsilon}\right)+\left(S_{A} \diamond f\left(2 \tau_{\varepsilon}+.\right)\right)\left(t-2 \tau_{\varepsilon}\right)
$$

and

$$
\begin{aligned}
& \left|\left(S_{A} \diamond f\right)(t)\right| \leq e^{\omega_{A}\left(t-2 \tau_{\varepsilon}\right)} \varepsilon_{1} e^{\gamma 2 \tau_{\varepsilon}}\left(e^{\left(\omega_{A}-\gamma\right) \tau_{\varepsilon}}+1\right) \\
& \quad \times \sup _{s \in\left[0,2 \tau_{\varepsilon}\right]} e^{-\gamma s}\|f(s)\|+\varepsilon \sup _{s \in\left[2 \tau_{\varepsilon}, t\right]}\|f(s)\| \\
& \leq e^{\omega_{A}\left(t-2 \tau_{\varepsilon}\right)} \varepsilon_{1} e^{\gamma 2 \tau_{\varepsilon}}\left(e^{\left(\omega_{A}-\gamma\right) \tau_{\varepsilon}}+1\right) \\
& \quad \times \sup _{s \in\left[0,2 \tau_{\varepsilon}\right]} e^{-\gamma s}\|f(s)\|+\varepsilon_{1} e^{\gamma t} \sup _{s \in\left[2 \tau_{\varepsilon}, t\right]} e^{-\gamma s}\|f(s)\| \\
& \leq \varepsilon_{1} e^{\gamma t}\left[e^{\left(\omega_{A}-\gamma\right)\left(t-2 \tau_{\varepsilon}\right)}\left(e^{\left(\omega_{A}-\gamma\right) \tau_{\varepsilon}}+1\right)+1\right] \sup _{s \in[0, t]} e^{-\gamma s}\|f(s)\| .
\end{aligned}
$$

By induction, we obtain for all $k \in \mathbb{N}$ with $k \geq 1, t \in\left[k \tau_{\varepsilon},(k+1) \tau_{\varepsilon}\right]$, and for each $\gamma>\omega_{A}$ that

$$
\left|\left(S_{A} \diamond f\right)(t)\right| \leq \varepsilon_{1} e^{\gamma t} \sup _{s \in[0, t]} e^{-\gamma s}\|f(s)\|\left[e^{\left(\omega_{A}-\gamma\right)\left(t-k \tau_{\varepsilon}\right)} \sum_{n=0}^{k-1}\left(e^{\left(\omega_{A}-\gamma\right) \tau_{\varepsilon}}\right)^{n}+1\right]
$$




$$
\leq \varepsilon_{1} e^{\gamma t} \sup _{s \in[0, t]} e^{-\gamma s}\|f(s)\|\left[\sum_{n=0}^{\infty}\left(e^{\left(\omega_{A}-\gamma\right) \tau_{\varepsilon}}\right)^{n}+1\right] .
$$

Since $\gamma>\omega_{A}$, we have for each $t \geq 0$ that

$$
e^{-\gamma t}\left\|\left(S_{A} \diamond f\right)(t)\right\| \leq e^{-\gamma t}\left|\left(S_{A} \diamond f\right)(t)\right| \leq \frac{2 \varepsilon_{1}}{1-e^{\left(\omega_{A}-\gamma\right) \tau_{\varepsilon}}} \sup _{s \in[0, t]} e^{-\gamma s}\|f(s)\| .
$$

This completes the proof.

Let $I \subset[0,+\infty)$ be an interval. Set $s:=\inf I \geq 0$. For each $\gamma \geq 0$, define

$$
B C^{\gamma}(I, Y):=\left\{\varphi \in C(I, Y): \sup _{l \in I} e^{-\gamma(l-s)}\|\varphi(l)\|_{Y}<+\infty\right\}
$$

and

$$
\|\varphi\|_{B C^{\gamma}(I, Y)}:=\sup _{l \in I} e^{-\gamma(l-s)}\|\varphi(l)\|_{Y} .
$$

It is well known that $B C^{\gamma}(I, Y)$ endowed with the norm $\|\cdot\|_{B C^{\gamma}(I, Y)}$ is a Banach space.

By using Proposition 2.14 we obtain the following result.

Lemma 2.15. Let Assumptions 2.1 and 2.8 be satisfied. For each $s \geq 0$ and each $\sigma \in(s,+\infty]$, define a linear operator $\mathcal{L}_{s}: C([s, \sigma), X) \rightarrow C\left([s, \sigma), X_{0}\right)$ by

$$
\mathcal{L}_{s}(\varphi)(t)=\left(S_{A} \diamond \varphi(.+s)\right)(t-s), \quad \forall t \in[s, \sigma), \quad \forall \varphi \in C([s, \sigma), X) .
$$

Then, for each $\gamma>\omega_{A}, \mathcal{L}_{s}$ is a bounded linear operator from $B C^{\gamma}([s, \sigma), X)$ into $B C^{\gamma}\left([s, \sigma), X_{0}\right)$. Moreover, for each $\varepsilon>0$ and each $\tau_{\varepsilon}>0$ such that $M_{A} V^{\infty}\left(S_{A}, 0, \tau_{\varepsilon}\right) \leq \varepsilon$,

$$
\left\|\mathcal{L}_{s}(\varphi)\right\|_{\mathcal{L}\left(B C \gamma([s, \sigma), X), B C \gamma\left([s, \sigma), X_{0}\right)\right)} \leq C(\varepsilon, \gamma) .
$$

Proof. Let $\varphi \in B C^{\gamma}([s, \sigma), X)$ be fixed. By using Proposition 2.14, we have for $t \in[s, \sigma)$ that

$$
\begin{aligned}
& e^{-\gamma(t-s)}\left\|\left(S_{A} \diamond \varphi(.+s)\right)(t-s)\right\| \leq C(\varepsilon, \gamma) \sup _{l \in[0, t-s]} e^{-\gamma l}\|\varphi(l+s)\| \\
& =C(\varepsilon, \gamma) \sup _{r \in[s, t]} e^{-\gamma(r-s)}\|\varphi(r)\| \leq C(\varepsilon, \gamma) \sup _{r \in[s, \sigma)} e^{-\gamma(r-s)}\|\varphi(r)\|
\end{aligned}
$$

and the result follows. 


\section{Semilinear Problems - Positivity}

Starting from this section, we consider the semilinear Cauchy problem

$$
\frac{d U(t, s) x}{d t}=A U(t, s) x+F(t, U(t, s) x), t \geq s, \quad U(s, s) x=x \in X_{0} .
$$

We shall investigate the properties of the non-autonomous semiflow generated by the following problem

$$
U(t, s) x=x+A \int_{s}^{t} U(l, s) x d l+\int_{s}^{t} F(l, U(l, s) x) d l, \quad t \geq s \geq 0,
$$

or equivalently

$$
U(t, s) x=T_{A_{0}}(t-s) x+\left(S_{A} \diamond F(.+s, U(.+s, s) x)\right)(t-s), \quad t \geq s \geq 0 .
$$

Definition 3.1. Consider two maps $\chi:[0,+\infty) \times X_{0} \rightarrow(0,+\infty]$ and $U$ : $D_{\chi} \rightarrow X_{0}$, where

$$
D_{\chi}=\left\{(t, s, x) \in[0,+\infty)^{2} \times X_{0}: s \leq t<s+\chi(s, x)\right\} .
$$

We say that $U$ is a maximal non-autonomous semiflow on $X_{0}$ if $U$ satisfies the following properties:

(i) $\chi(r, U(r, s) x)+r=\chi(s, x)+s, \forall s \geq 0, \forall x \in X_{0}, \forall r \in[s, s+\chi(s, x))$.

(ii) $U(s, s) x=x, \forall s \geq 0, \forall x \in X_{0}$.

(iii) $U(t, r) U(r, s) x=U(t, s) x, \forall s \geq 0, \forall x \in X_{0}, \forall t, r \in[s, s+\chi(s, x))$ with $t \geq r$.

(iv) If $\chi(s, x)<+\infty$, then

$$
\lim _{t \rightarrow(s+\chi(s, x))^{-}}\|U(t, s) x\|=+\infty .
$$

Set

$$
D=\left\{(t, s, x) \in[0,+\infty)^{2} \times X_{0}: t \geq s\right\} .
$$

In order to present a theorem on the existence and uniqueness of solutions to equation (3.1), we make the following assumption.

Assumption 3.2. Assume that $F:[0,+\infty) \times \overline{D(A)} \rightarrow X$ is a continuous map, and for each $\sigma>0$ and each $\xi>0$ there exists $K(\sigma, \xi)>0$ such that

$$
\|F(t, x)-F(t, y)\| \leq K(\sigma, \xi)\|x-y\|
$$

whenever $t \in[0, \sigma], y, x \in X_{0}$ with $\|x\| \leq \xi$ and $\|y\| \leq \xi$.

The following theorem is proved in Magal and Ruan [22, Theorem 5.2]. 
Theorem 3.3. Let Assumptions 2.1-2.8 and 3.2 be satisfied. Then there exist a map $\chi:[0,+\infty) \times X_{0} \rightarrow(0,+\infty]$ and a maximal non-autonomous semiflow $U: D_{\chi} \rightarrow X_{0}$, such that for each $x \in X_{0}$ and each $s \geq 0, U(., s) x \in$ $C\left([s, s+\chi(s, x)), X_{0}\right)$ is a unique maximal solution of (3.1) (or equivalently a unique maximal solution of (3.2)). Moreover, $D_{\chi}$ is open in $D$ and the map $(t, s, x) \rightarrow U(t, s) x$ is continuous from $D_{\chi}$ into $X_{0}$.

We are now interested in the positivity of the solutions of equation (3.1). Let $X_{+} \subset X$ be a cone of $X$; that is, $X_{+}$is a closed convex subset of $X$ satisfying the following two properties:

(i) $\lambda x \in X_{+}, \forall x \in X_{+}, \forall \lambda \geq 0$.

(ii) $x \in X_{+}$and $-x \in X_{+} \Rightarrow x=0$.

It is clear that $X_{0+}=X_{0} \cap X_{+}$is also a cone of $X_{0}$. Recall that such a cone defines a partial order on the Banach space $X$ which is defined by

$$
x \geq y \quad \text { if and only if } \quad x-y \in X_{+} .
$$

We need the following assumption to prove the positivity of solutions of equation (3.1).

Assumption 3.4. Assume that there exists a linear operator $B \in \mathcal{L}\left(X_{0}, X\right)$ such that

(a) for each $\gamma>0, A-\gamma B$ is resolvent positive (i.e., $(\lambda I-(A-\gamma B))^{-1} X_{+}$ $\subset X_{+}$for all $\lambda>\omega_{A}$ large enough);

(b) for each $\xi>0$ and each $\sigma>0$, there exists $\gamma=\gamma(\xi, \sigma)>0$, such that $F(t, x)+\gamma B x \in X_{+}$whenever $x \in X_{0+},\|x\| \leq \xi$ and $t \in[0, \sigma]$.

Proposition 3.5. Let Assumptions 2.1-2.8 and 3.2-3.4 be satisfied. Then for each $x \in X_{0+}$ and each $s \geq 0$, we have $U(t, s) x \in X_{0+}$ for all $t \in$ $[s, s+\chi(s, x))$.

Proof. Without loss of generality we can assume that $s=0$ and $x \in X_{0+}$. Moreover, using the semiflow property, it is sufficient to prove that there exists $\varepsilon \in(0, \chi(0, x))$ such that $U(t, 0) x \in X_{0+}$ for all $t \in[0, \varepsilon]$. Let $x \in X_{0+}$ be fixed. We set $\xi:=2(\|x\|+1)$. Let $\gamma>0$ such that $F(t, x)+\gamma B x \in X_{+}$ when $x \in X_{0+},\|x\| \leq \xi$ and $t \in[0,1]$. Fix $\tau_{\gamma}>0$ such that $\gamma V^{\infty}\left(S_{A}, 0, \tau_{\gamma}\right)<$ 1. For each $\sigma \in\left(0, \tau_{\gamma}\right)$, define

$$
E^{\sigma}=\left\{\varphi \in C\left([0, \sigma], X_{0+}\right):\|\varphi(t)\| \leq \xi, \forall t \in[0, \sigma]\right\} .
$$

Then it is sufficient to consider the fixed-point problem

$u(t)=T_{(A-\gamma B)_{0}}(t) x+\left(S_{A-\gamma B} \diamond F(., u())+.\gamma B u().\right)(t)=: \Psi(u)(t), \forall t \in[0, \sigma]$. 
Since $A-\gamma B$ is resolvent positive, we have $T_{(A-\gamma B)_{0}}(t) X_{0+} \subset X_{0+}$ for all $t \geq 0$. Using the approximation formula (2.7), we have for each $\tau>0$ that

$$
\left(S_{A-\gamma B} \diamond \varphi\right)(t) \in X_{0+}, \quad \forall t \in[0, \tau], \forall \varphi \in C\left([0, \tau], X_{+}\right) .
$$

Moreover, by using Theorem 2.12, for each $\varphi \in E^{\sigma}$ and each $t \in[0, \sigma]$, we deduce that

$$
\begin{aligned}
& \|\Psi(\varphi)(t)\|=\left\|T_{(A-\gamma B)_{0}}(t) x+\left(S_{A-\gamma B} \diamond F(., \varphi(.))+\gamma \varphi(.)\right)(t)\right\| \\
& \leq\left\|T_{(A-\gamma B)_{0}}(t) x\right\|+\frac{V^{\infty}\left(S_{A}, 0, t\right)}{1-\gamma V^{\infty}\left(S_{A}, 0, \tau_{\gamma}\right)} \sup _{s \in[0, t]}\|F(s, \varphi(s))+\gamma \varphi(s)\| \\
& \leq \sup _{t \in[0, \sigma]}\left\|T_{(A-\gamma B)_{0}}(t) x\right\| \\
& \quad+\frac{V^{\infty}\left(S_{A}, 0, \sigma\right)}{1-\gamma V^{\infty}\left(S_{A}, 0, \tau_{\gamma}\right)}\left[\sup _{s \in[0, \sigma]}\|F(s, 0)\|+[K(1, \xi)+\gamma] \xi\right] .
\end{aligned}
$$

Hence, there exists $\sigma_{1} \in(0,1)$ such that $\Psi\left(E^{\sigma}\right) \subset E^{\sigma}$ for all $\sigma \in\left(0, \sigma_{1}\right]$. Therefore, for each $\sigma \in\left(0, \sigma_{1}\right]$ and each pair $\varphi, \psi \in E^{\sigma}$, we have for $t \in[0, \sigma]$ that

$$
\begin{aligned}
& \|\Psi(\varphi)(t)-\Psi(\psi)(t)\|=\left\|\left(S_{A-\gamma B} \diamond[F(., \varphi(.))-F(., \psi(.))+\gamma(\varphi-\psi)(.)]\right)(t)\right\| \\
& \leq \frac{V^{\infty}\left(S_{A}, 0, \sigma\right)}{1-\gamma V^{\infty}\left(S_{A}, 0, \tau_{\gamma}\right)}[K(1, \xi)+\gamma] \sup _{s \in[0, \sigma]}\|(\varphi-\psi)(s)\| .
\end{aligned}
$$

Thus, there exists $\sigma_{2} \in\left(0, \sigma_{1}\right]$ such that $\Psi\left(E^{\sigma_{2}}\right) \subset E^{\sigma_{2}}$ and $\Psi$ is a strict contraction on $E^{\sigma_{2}}$. The result then follows.

Example 3.6. (1) We refer to Thieme [35] and Hirsch and Smith [17] for more results on the positivity of semiflows.

(2) Usually Proposition 3.5 is applied with $B=I$. But the case $B \neq I$ can also be useful. Consider the following functional differential equation:

$$
\left\{\begin{array}{l}
\frac{d x(t)}{d t}=f\left(x_{t}\right), \forall t \geq 0, \\
x_{0}=\varphi \in C\left([-\tau, 0], \mathbb{R}^{n}\right),
\end{array}\right.
$$

where $f: C\left([-\tau, 0], \mathbb{R}^{n}\right) \rightarrow \mathbb{R}^{n}$ is Lipschitz continuous on bounded subsets of $C\left([-\tau, 0], \mathbb{R}^{n}\right)$. In order to obtain the positivity of solutions, it is sufficient to assume that for each $M \geq 0$ there exists $\gamma=\gamma(M)>0$ such that

$$
f(\varphi)+\gamma \varphi(0) \geq 0
$$

whenever $\|\varphi\|_{\infty} \leq M$ and $\varphi \in C\left([-\tau, 0], \mathbb{R}_{+}^{n}\right)$. It is well known that this condition is sufficient to obtain the positivity of solutions (see Martin and 
Smith $[26,27])$. In order to prove this, one may also apply Proposition 3.5. By identifying $x_{t}$ with $u(t)=\left(\begin{array}{c}0 \\ x_{t}\end{array}\right)$, the system (3.3) can be rewritten as a non-densely defined Cauchy problem (see Liu, Magal and Ruan [19] for more details)

$$
\frac{d v(t)}{d t}=A v(t)+F(v(t)), \forall t \geq 0, \text { and } v(0)=\left(\begin{array}{c}
0 \\
\varphi
\end{array}\right)
$$

with $X=\mathbb{R}^{n} \times C\left([-\tau, 0], \mathbb{R}^{n}\right), D(A)=\left\{0_{\mathbb{R}^{n}}\right\} \times C^{1}\left([-\tau, 0], \mathbb{R}^{n}\right)$, where $A: D(A) \subset X \rightarrow X$ is defined by

$$
A\left(\begin{array}{c}
0 \\
\varphi
\end{array}\right)=\left(\begin{array}{c}
-\varphi^{\prime}(0) \\
\varphi^{\prime}
\end{array}\right)
$$

and $F: \overline{D(A)} \rightarrow X$ by

$$
F\left(\begin{array}{l}
0 \\
\varphi
\end{array}\right)=\left(\begin{array}{c}
f(\varphi) \\
0_{C}
\end{array}\right)
$$

Then Proposition 3.5 applies with

$$
B\left(\begin{array}{l}
0 \\
\varphi
\end{array}\right)=\left(\begin{array}{c}
\varphi(0) \\
0_{C}
\end{array}\right)
$$

Recall that a cone $X_{+}$of a Banach space $(X,\|\cdot\|)$ is normal if there exists a norm $\|.\|_{1}$ equivalent to $\|$.$\| , which is monotone; that is,$

$$
\forall x, y \in X, 0 \leq x \leq y \Rightarrow\|x\|_{1} \leq\|y\|_{1} .
$$

Corollary 3.7. Let Assumptions 2.1-2.8 and 3.2-3.4 be satisfied. Assume in addition that

(a) $X_{+}$is a normal cone of $(X,\|\cdot\|)$;

(b) there exist a continuous map $G:[0,+\infty) \times X_{0+} \rightarrow X_{+}$and two real numbers $k_{1} \geq 0$ and $k_{2} \geq 0$, such that for each $t \geq 0$ and each $x \in X_{0+}$,

$$
F(t, x) \leq G(t, x) \text { and }\|G(t, x)\| \leq k_{1}\|x\|+k_{2} .
$$

Then

$$
\chi(s, x)=+\infty, \quad \forall s \geq 0, \quad \forall x \in X_{0+} .
$$

Moreover, we have the following estimate: for each $\gamma>0$ large enough, there exist $C_{1}>0$ and $C_{2}>0$ such that

$$
\|U(t, s) x\| \leq e^{\gamma(t-s)}\left[C_{1}\|x\|+C_{2}\right] .
$$


Proof. Without loss of generality, we can assume that $s=0$ and the norm $\|$.$\| is monotone. Let \varepsilon \in\left(0, \frac{1}{2 k_{1}}\right)$ and $\tau_{\varepsilon}>0$ such that $M_{A} V^{\infty}\left(S_{A}, 0, \tau_{\varepsilon}\right) \leq$ $\varepsilon$. Let $x \in X_{0+}$ be fixed. Then by Proposition 3.5, we have for each $t \in$ $[0, \chi(0, x))$ that

$$
\begin{aligned}
0 & \leq U(t, 0) x=T_{A_{0}}(t) x+\left(S_{A} \diamond F(., U(., 0) x)\right)(t) \\
& \leq T_{A_{0}}(t) x+\left(S_{A} \diamond G(., U(., 0) x)\right)(t) .
\end{aligned}
$$

Hence, for each $\gamma>\max \left(\omega_{A}, 0\right)$, we have for each $t \in[0, \chi(0, x))$ that

$$
\begin{aligned}
e^{-\gamma t}\|U(t, 0) x\| & \leq e^{-\gamma t}\left\|T_{A_{0}}(t) x\right\|+e^{-\gamma t}\left\|\left(S_{A} \diamond G(., U(., 0) x)\right)(t)\right\| \\
& \leq M_{A} e^{\left(-\gamma+\omega_{A}\right) t}\|x\|+C(\varepsilon, \gamma) \sup _{s \in[0, t]} e^{-\gamma s}\|G(s, U(s, 0) x)\| \\
& \leq M_{A}\|x\|+C(\varepsilon, \gamma) \sup _{s \in[0, t]} e^{-\gamma s}\left[k_{1}\|U(s, 0) x\|+k_{2}\right] \\
& \leq M_{A}\|x\|+k_{2} C(\varepsilon, \gamma)+k_{1} C(\varepsilon, \gamma) \sup _{s \in[0, t]} e^{-\gamma s}\|U(s, 0) x\| .
\end{aligned}
$$

Since $2 k_{1} \varepsilon<1$, for $\gamma>\max \left(\omega_{A}, 0\right)$ sufficiently large, we obtain $k_{1} C(\varepsilon, \gamma)=$ $\frac{2 k_{1} \varepsilon}{1-e^{\left(\omega_{A}-\gamma\right) \tau_{\varepsilon}}}<1$ and the result follows.

\section{Global Lipschitz Perturbation}

We now consider the case where the map $x \rightarrow F(t, x)$ is Lipschitz continuous. Let $\left(Y,\|\cdot\|_{Y}\right)$ and $\left(Z,\|\cdot\|_{Z}\right)$ be two Banach spaces. Let $E$ be a subset of $Y$ and $G: Y \rightarrow Z$ be a map. Define

$$
\|G\|_{\operatorname{Lip}(E, Z)}=\sup _{x, y \in E: x \neq y} \frac{\|G(x)-G(y)\|_{Z}}{\|x-y\|_{Y}} .
$$

For each $x \in Y$ and $r>0$, set

$$
B_{Y}(x, r)=\left\{y \in Y:\|x-y\|_{Y}<r\right\}, \bar{B}_{Y}(x, r)=\left\{y \in Y:\|x-y\|_{Y} \leq r\right\} .
$$

The main results of this section are on the existence and uniqueness of a solution to the integral equation (3.1) and its estimate when $x \rightarrow F(t, x) x$ is globally Lipschitz continuous.

Proposition 4.1. Let Assumptions 2.1-2.8 be satisfied. Let $F:[0,+\infty) \times$ $\overline{D(A)} \rightarrow X$ be a continuous map and $\sigma \in(0,+\infty]$ be a fixed constant. Assume that

$$
\Gamma_{F}(\sigma):=\sup _{t \in[0, \sigma)}\|F(t, .)\|_{\operatorname{Lip}\left(X_{0}, X\right)}<+\infty
$$


Then, for each $x \in X_{0}$ and each $s \in[0, \sigma)$, there exists a unique solution $U(., s) x \in C\left([s, \sigma), X_{0}\right)$ of

$$
U(t, s) x=x+A \int_{s}^{t} U(l, s) x d l+\int_{s}^{t} F(l, U(l, s) x) d l, \quad \forall t \in[s, \sigma) .
$$

Moreover, there exists $\gamma_{0}>\max \left(0, \omega_{A}\right)$ such that for each $\gamma \geq \gamma_{0}$, each pair $t, s \in[0, \sigma)$ with $t \geq s$, and each pair $x, y \in X_{0}$, we have

$$
\|U(t, s) x\| \leq e^{\gamma(t-s)}\left[2 M_{A}\|x\|+\sup _{l \in[s, \sigma)} e^{-\gamma(l-s)}\|F(l, 0)\|\right]
$$

and

$$
\|U(t, s) x-U(t, s) y\| \leq e^{\gamma(t-s)} 2 M_{A}\|x-y\| .
$$

Proof. Fix $s, t \in[0, \sigma)$ with $s<t$. Let $\varepsilon>0$ such that

$$
\varepsilon \max \left(\Gamma_{F}(\sigma), 1\right)<1 / 8 \text {. }
$$

Let $\tau_{\varepsilon}>0$ such that $M_{A} V^{\infty}\left(S_{A}, 0, \tau_{\varepsilon}\right) \leq \varepsilon$. Then by Lemma 2.15 we have for each $\gamma>\omega_{A}$ that

$$
\left\|\mathcal{L}_{s}(\varphi)\right\|_{\mathcal{L}\left(B C^{\gamma}([s,+\infty), X), B C^{\gamma}\left([s,+\infty), X_{0}\right)\right)} \leq C(\gamma, \varepsilon)=\frac{2 \varepsilon \max \left(1, e^{-\gamma \tau_{\varepsilon}}\right)}{\left(1-e^{\left(\omega_{A}-\gamma\right) \tau_{\varepsilon}}\right)}
$$

Let $\gamma_{0} \geq \max \left(0, \omega_{A}\right)$ such that

$$
\frac{1}{\left(1-e^{\left.\left(\omega_{A}-\gamma\right) \tau_{\varepsilon}\right)}\right.}<2, \quad \forall \gamma \geq \gamma_{0}
$$

To prove the proposition it is sufficient to prove that the following fixed-point problem

$$
U(., s) x=T_{A_{0}}(.-s) x+\mathcal{L}_{s} \circ \Psi(U(., s) x)
$$

admits a solution $U(., s) x \in B C^{\gamma}\left([s, \sigma), X_{0}\right)$, where $\Psi: B C^{\gamma}\left([s, \sigma), X_{0}\right) \rightarrow$ $B C^{\gamma}([s, \sigma), X)$ is a nonlinear operator defined by

$$
\Psi(\varphi)(l)=F(l, \varphi(l)), \quad \forall l \in[s, t], \forall \varphi \in B C^{\gamma}\left([s, \sigma), X_{0}\right) .
$$

We have

and

$$
\begin{gathered}
\left\|T_{A_{0}}(.-s)\right\|_{\mathcal{L}\left(X_{0}, B C^{\gamma}\left([s, \sigma), X_{0}\right)\right)} \leq M_{A}, \\
\left\|\mathcal{L}_{s}\right\|_{\mathcal{L}\left(B C^{\gamma}([s, \sigma), X), B C^{\gamma}\left([s, \sigma), X_{0}\right)\right)} \leq 4 \varepsilon,
\end{gathered}
$$

From this we deduce that

$$
\|\Psi\|_{\operatorname{Lip}\left(B C^{\gamma}\left([s, \sigma), X_{0}\right), B C^{\gamma}([s, \sigma), X)\right)} \leq \Gamma_{F}(\sigma) .
$$

$$
\left\|\mathcal{L}_{s} \circ \Psi\right\|_{\operatorname{Lip}\left(B C^{\gamma}\left([s, \sigma), X_{0}\right), B C^{\gamma}\left([s, \sigma), X_{0}\right)\right)} \leq 4 \varepsilon \Gamma_{F}(\sigma) \leq 1 / 2 .
$$


Thus, the fixed-point problem (3.4) has a unique solution. Moreover, for each $x \in X_{0}$, there exists a unique solution $U(., s) x$ in $B C^{\gamma}\left([s, \sigma), X_{0}\right)$ such that

$$
\begin{aligned}
& \|U(., s) x\|_{B C^{\gamma}\left([s, t], X_{0}\right)} \\
& \quad \leq M_{A}\|x\|+\left\|\mathcal{L}_{s}(\Psi(0))\right\|+\left\|\mathcal{L}_{s}(\Psi(U(., s) x)-\Psi(0))\right\| \\
& \quad \leq M_{A}\|x\|+4 \varepsilon\|\Psi(0)\|_{B C \gamma([s, \sigma), X)}+\frac{1}{2}\|U(., s) x\|_{B C \gamma\left([s, \sigma), X_{0}\right)},
\end{aligned}
$$

which implies that

$$
\|U(., s) x\|_{B C^{\gamma}\left([s, \sigma), X_{0}\right)} \leq 2 M_{A}\|x\|+8 \varepsilon\|\Psi(0)\|_{B C^{\gamma}([s, \sigma), X)} .
$$

Since by construction $\varepsilon \leq \frac{1}{8}$, we have

$$
\sup _{l \in[s, \sigma)} e^{-\gamma(l-s)}\|U(., s) x\| \leq 2 M_{A}\|x\|+\sup _{l \in[s, \sigma)} e^{-\gamma(l-s)}\|F(l, 0)\| .
$$

Similarly, we have for each pair $x, y \in X_{0}$ that

$$
U(., s) x-U(., s) y=T_{A_{0}}(.-s)(x-y)+\mathcal{L}_{s}[\Psi(U(., s) x)-\Psi(U(., s) y)] .
$$

Therefore,

$$
\begin{aligned}
& \|U(., s) x-U(., s) y\|_{B C^{\gamma}\left([s, \sigma), X_{0}\right)} \\
& \quad \leq M_{A}\|x-y\|+\frac{1}{2}\|U(., s) x-U(., s) y\|_{B C^{\gamma}\left([s, \sigma), X_{0}\right)} .
\end{aligned}
$$

This completes the proof.

\section{Differentiability with Respect to the State Variable}

In this section we investigate the differentiability of solutions with respect to the state variable.

Proposition 5.1. Let Assumptions 2.1-2.8 and 3.2 be satisfied. Assume in addition that

(a) for each $t \geq 0$ the map $x \rightarrow F(t, x)$ is continuously differentiable from $X_{0}$ into $X$;

(b) the map $(t, x) \rightarrow D_{x} F(t, x)$ is continuous from $[0,+\infty) \times X_{0}$ into $\mathcal{L}\left(X_{0}, X\right)$.

Let $x_{0} \in X_{0}, s \geq 0, \tau \in\left[0, \chi\left(s, x_{0}\right)\right)$, and $\gamma \in\left(0, \chi\left(s, x_{0}\right)-\tau\right)$. Let $\eta>0$ (there exists such a constant since $D_{\chi}$ is open in $\left.D\right)$ such that

$$
\chi(s, y)>\tau+\gamma, \forall y \in B_{X_{0}}\left(x_{0}, \eta\right) .
$$


Then for each $t \in[s, s+\tau+\gamma]$, the map $x \rightarrow U(t, s) x$ is defined from $B_{X_{0}}(x, \eta)$ into $X_{0}$ and is differentiable at $x_{0}$. Moreover, if we set

$$
V(t, s) y=D_{x} U(t, s)(x)(y), \forall y \in X_{0},
$$

then $t \rightarrow V(t, s) y$ is an integrated solution of the Cauchy problem

$$
\begin{aligned}
\frac{d V(t, s) y}{d t} & =A V(t, s) y+D_{x} F\left(t, U(t, s) x_{0}\right) V(t, s) y, t \in\left[s, s+\chi\left(s, x_{0}\right)\right), \\
V(s, s) y & =y
\end{aligned}
$$

or equivalently, for all $t \in\left[s, s+\chi\left(s, x_{0}\right)\right), t \rightarrow V(t, s) y$ is a solution of

$$
V(t, s) y=T_{A_{0}}(t-s) y+\left(S_{A} \diamond D_{x} F\left(., U(., s) x_{0}\right) V(., s) y\right)(t-s) .
$$

Proof. First by using the result in Section 4 about the Lipschitz case, it clear that $W(t, s)$ is well defined. Set

$$
R(t)(y)=U(t, s)\left(x_{0}+y\right)-U(t, s)\left(x_{0}\right)-V(t, s) y .
$$

Then

$$
\begin{aligned}
R(t)(y)= & \left(S _ { A } \diamond \left[F\left(., U(., s)\left(x_{0}+y\right)\right)-F\left(., U(., s)\left(x_{0}\right)\right)\right.\right. \\
& \left.\left.-D_{x} F\left(., U(., s) x_{0}\right) V(., s) y\right]\right)(t-s) .
\end{aligned}
$$

But

$$
\begin{aligned}
F( & \left.t, U(t, s)\left(x_{0}+y\right)\right)-F\left(t, U(t, s)\left(x_{0}\right)\right) \\
= & \int_{0}^{1} D_{x} F\left(t, r U(t, s)\left(x_{0}+y\right)+(1-r) U(t, s)\left(x_{0}\right)\right)\left(U(t, s)\left(x_{0}+y\right)\right. \\
& \left.-U(t, s)\left(x_{0}\right)\right) d r \\
= & \int_{0}^{1} \Psi_{1}(t, r, y)\left(U(t, s)\left(x_{0}+y\right)-U(t, s)\left(x_{0}\right)\right) d r \\
& +D_{x} F\left(t, U(t, s)\left(x_{0}\right)\right)\left(U(t, s)\left(x_{0}+y\right)-U(t, s)\left(x_{0}\right)\right),
\end{aligned}
$$

where

$$
\Psi_{1}(t, r, y)=D_{x} F\left(t, r U(t, s)\left(x_{0}+y\right)+(1-r) U(t, s)\left(x_{0}\right)\right)-D_{x} F\left(t, U(t, s)\left(x_{0}\right)\right) \text {. }
$$

Thus,

$$
R(t) y=\left(S_{A} \diamond\left[K(.)+D_{x} F\left(., U(., s) x_{0}\right) R(.) y\right]\right)(t-s)
$$

where

and

$$
K(t)=\int_{0}^{1} \Psi_{2}(t, r, y)\left(U(t, s)\left(x_{0}+y\right)-U(t, s)\left(x_{0}\right)\right) d r
$$

$\Psi_{2}(t, r, y)=D_{x} F\left(t, r U(t, s)\left(x_{0}+y\right)+(1-r) U(t, s)\left(x_{0}\right)\right)-D_{x} F\left(t, U(t, s)\left(x_{0}\right)\right)$. 
The result follows from Proposition 2.14 and the continuity of $(t, x) \rightarrow$ $U(t, s) x$.

\section{Time Differentiability}

In this section, we study the time differentiability of the solutions. Consider a solution $u \in C([0, \tau], \overline{D(A)})$ of

$$
u(t)=x_{0}+A \int_{0}^{t} u(s) d s+\int_{0}^{t} F(s, u(s)) d s, t \in[0, \tau] .
$$

Assume that $x_{0} \in D(A)$ and $F:[0, T] \times \overline{D(A)} \rightarrow X$ is a $C^{1}$ map. When the domain of $A$ is dense, it is well known (see Pazy [30], Theorem 6.1.5, page 187) that, for each $x \in D(A)$, the map $t \rightarrow u(t)$ is a classical solution. That is, the map $t \rightarrow u(t)$ is continuously differentiable and $u(t) \in D(A)$ for all $t \in[0, \tau]$, and satisfies

$$
u^{\prime}(t)=A u(t)+f(t, u(t)), \forall t \in[0, \tau], \quad u(0)=x_{0} .
$$

In this section, we consider the same problem but in the the context of non-densely defined Cauchy problems. When $A$ satisfies the Hille-Yosida condition, this problem has been studied by Thieme [35] and Magal [21]. So the goal is to extend these results to the non-Hille-Yosida case. This problem turns out to be more difficult. For each $\tau>0$, set

$$
C^{1,+}([0, \tau], X)=\left\{f \in C([0, \tau], X): \frac{d^{+} f}{d t} \in C([0, \tau), X), \lim _{t / \tau} \frac{d^{+} f}{d t}(t)<\infty\right\} .
$$

The following lemma is a variant of a result due to Da Prato and Sinestrari [7].

Lemma 6.1. Let $A: D(A) \subset X \rightarrow X$ be a closed linear operator. Let $\tau>0, f \in C([0, \tau], X)$, and $x \in X_{0}$ be fixed. Assume that $u \in C([0, \tau], X)$ is a solution of

$$
u(t)=x+A \int_{0}^{t} u(s) d s+\int_{0}^{t} f(s) d s, \forall t \in[0, \tau] .
$$

Assume in addition that $u$ belongs to $C^{1,+}([0, \tau], X)$ or $C([0, \tau], D(A))$. Then $u \in C^{1}([0, \tau], X) \cap C([0, \tau], D(A))$ and

$$
u^{\prime}(t)=A u(t)+f(t), \quad \forall t \in[0, \tau] .
$$

Proof. If $u \in C([0, T], D(A))$, since $A$ is closed, we have

$$
u(t)=x+\int_{0}^{t} A u(s) d s+\int_{0}^{t} f(s) d s, \quad \forall t \in[0, \tau] .
$$


Thus $u \in C^{1}([0, \tau], X)$ and $u^{\prime}(t)=A u(t)+f(t), \forall t \in[0, \tau]$.

If $u \in C^{1,+}([0, \tau], X)$, then we have for each $t \in[0, \tau)$ and $h>0$ that

$$
\frac{u(t+h)-u(t)}{h}-\frac{\int_{t}^{t+h} f(s) d s}{h}=A \frac{\int_{t}^{t+h} u(s) d s}{h} .
$$

Since $A$ is closed, we deduce that $u(t) \in D(A)$ and $A u(t)=\frac{d^{+} u}{d t}(t)+f(t)$ for all $t \in[0, \tau)$. Since $u \in C^{1,+}([0, \tau], X)$, we then deduce that $u \in$ $C([0, \tau], D(A))$ and complete the proof.

Lemma 6.2. Let Assumptions 2.1 and 2.8 be satisfied. Assume that $g \in$ $C^{1}([0, T], X)$ and $g(0) \in \overline{D(A)}$; then $t \rightarrow\left(S_{A} \diamond g\right)(t)$ is continuously differentiable and

$$
\frac{d}{d t}\left(S_{A} \diamond g\right)(t)=T_{A_{0}}(t) g(0)+\left(S_{A} \diamond g^{\prime}\right)(t), \quad \forall t \in[0, T] .
$$

Proof. Since $g$ is continuously differentiable, the map $t \rightarrow\left(S_{A} * g\right)(t)$ is continuously differentiable, with

$$
\frac{d}{d t}\left(S_{A} * g\right)(t)=S_{A}(t) g(0)+\left(S_{A} * g^{\prime}\right)(t), \forall t \in[0, T] .
$$

Since $g(0) \in \overline{D(A)}$, we have $S_{A}(t) g(0)=\int_{0}^{t} T_{A_{0}}(l) g(0) d l$ for all $t \in[0, T]$, and the result follows.

The following theorem is due to Vanderbauwhede [39, Theorem 3.5].

Lemma 6.3. (Fibre contraction theorem) Let $M_{1}$ and $M_{2}$ be two complete metric spaces and $\Psi: M_{1} \times M_{2} \rightarrow M_{1} \times M_{2}$ a mapping of the form

$$
\Psi(x, y)=\left(\Psi_{1}(x), \Psi_{2}(x, y)\right), \forall(x, y) \in M_{1} \times M_{2}
$$

satisfying the following properties:

(i) $\Psi_{1}$ has a fixed point $\bar{x} \in M_{1}$ such that for each $x \in M_{1}$,

$$
\Psi_{1}^{n}(x) \rightarrow \bar{x} \text { as } n \rightarrow+\infty .
$$

(ii) There exists $k \in[0,1)$ such that for each $x \in M_{1}$ the map $y \rightarrow$ $\Psi_{2}(x, y)$ is k-Lipschitz continuous.

(iii) The map $x \rightarrow \Psi_{2}(x, \bar{y})$ is continuous, where $\bar{y} \in M_{2}$ is a fixed point of the map $y \rightarrow \Psi_{2}(\bar{x}, y)$.

Then, for each $(x, y) \in M_{1} \times M_{2}$,

$$
\Psi^{n}(x, y) \rightarrow(\bar{x}, \bar{y}) \text { as } n \rightarrow+\infty .
$$

The key result of this section is the following lemma. 
Lemma 6.4. Let Assumptions 2.1 and 2.8 be satisfied. Let $\tau>0$ be fixed and $F:[0, \tau] \times \overline{D(A)} \rightarrow X$ be continuously differentiable. Assume that there exists an integrated solution $u \in C([0, \tau], X)$ of the Cauchy problem

$$
\frac{d u(t)}{d t}=A u(t)+F(t, u(t)), t \in[0, \tau], u(0)=x \in X_{0} .
$$

Assume in addition that $x \in D\left(A_{0}\right)$ and $F(0, x) \in \overline{D(A)}$. Then there exists $\varepsilon>0$, such that $u \in C^{1}([0, \varepsilon], X) \cap C([0, \varepsilon], D(A))$ and

$$
u^{\prime}(t)=A u(t)+F(t, u(t)), \quad \forall t \in[0, \varepsilon] .
$$

Proof. Since $F$ is continuously differentiable, there exist $\varepsilon_{0}>0, K_{1}>0$, and $K_{2}>0$ such that

$$
\left\|\partial_{t} F(t, y)\right\| \leq K_{1} \text { and }\left\|\partial_{x} F(t, y)\right\|_{\mathcal{L}\left(X_{0}, X\right)} \leq K_{2}
$$

whenever $\|x-y\| \leq \varepsilon_{0}$ and $0 \leq t \leq \varepsilon_{0}$. For each $\varepsilon \in\left(0, \varepsilon_{0}\right]$, set

$$
\begin{gathered}
M_{1}^{\varepsilon}=\left\{\varphi \in C\left([0, \varepsilon], X_{0}\right): \varphi(0)=x,\|\varphi(t)-x\| \leq \varepsilon_{0}, \forall t \in[0, \varepsilon]\right\}, \\
M_{2}^{\varepsilon}=\left\{\varphi \in C\left([0, \varepsilon], X_{0}\right): \varphi(0)=A_{0} x+F(0, x),\right. \\
\left.\left\|\varphi(t)-A_{0} x+F(0, x)\right\| \leq \varepsilon_{0}, \forall t \in[0, \varepsilon]\right\} .
\end{gathered}
$$

From now on, we assume that for each $i=1,2, M_{i}^{\varepsilon}$ is endowed with the metric $d(\varphi, \widehat{\varphi})=\|\varphi-\widehat{\varphi}\|_{\infty,[0, \varepsilon]}$ and $M_{1}^{\varepsilon} \times M_{2}^{\varepsilon}$ is endowed with the usual product distance $d((\varphi, \psi),(\widehat{\varphi}, \widehat{\psi}))=d(\varphi, \widehat{\varphi})+d(\psi, \widehat{\psi})$.

For each $\varepsilon \in\left(0, \varepsilon_{0}\right]$, set

$$
E^{\varepsilon}=\left\{\left(\varphi_{1}, \varphi_{2}\right) \in M_{1}^{\varepsilon} \times M_{2}^{\varepsilon}: \varphi_{1}(t)=x+\int_{0}^{t} \varphi_{2}(s) d s, \forall t \in[0, \varepsilon]\right\} .
$$

Then it is clear that $E^{\varepsilon}$ is a closed subset of $M_{1}^{\varepsilon} \times M_{2}^{\varepsilon}$.

We consider a map $\Psi: M_{1}^{\varepsilon} \times M_{2}^{\varepsilon} \rightarrow C\left([0, \varepsilon], X_{0}\right) \times C\left([0, \varepsilon], X_{0}\right)$ defined by

$$
\Psi\left(\varphi_{1}, \varphi_{2}\right)=\left(\Psi_{1}\left(\varphi_{1}\right), \Psi_{2}\left(\varphi_{1}, \varphi_{2}\right)\right), \quad \forall\left(\varphi_{1}, \varphi_{2}\right) \in M_{1}^{\varepsilon} \times M_{2}^{\varepsilon},
$$

where, for each $t \in[0, \varepsilon]$,

$$
\begin{aligned}
\Psi_{1}\left(\varphi_{1}\right)(t)= & T_{A_{0}}(t) x+\left(S_{A} \diamond F\left(., \varphi_{1}(.)\right)\right)(t), \\
\Psi_{2}\left(\varphi_{1}, \varphi_{2}\right)(t)= & T_{A_{0}}(t)\left[A_{0} x+F(0, x)\right] \\
& +\left(S_{A} \diamond \partial_{t} F\left(., \varphi_{1}(.)\right)+\partial_{x} F\left(., \varphi_{1}(.)\right) \varphi_{2}(.)\right)(t) .
\end{aligned}
$$

One can easily check that $\Psi$ is a continuous map. We now prove that for some $\varepsilon>0$ small enough, $\Psi\left(M_{1}^{\varepsilon} \times M_{2}^{\varepsilon}\right) \subset M_{1}^{\varepsilon} \times M_{2}^{\varepsilon}$, and

$$
\Psi_{1}\left(\varphi_{1}\right)(0)=x, \quad \Psi_{2}\left(\varphi_{1}, \varphi_{2}\right)(0)=\left[A_{0} x+F(0, x)\right] .
$$


For each $\varepsilon \in\left(0, \varepsilon_{0}\right]$, each $t \in[0, \varepsilon]$, and each $\varphi \in M_{1}^{\varepsilon}$, we have

$$
\begin{aligned}
& \left\|\Psi_{1}(\varphi)(t)-x\right\| \leq\left\|T_{A_{0}}(t) x-x\right\|+\|\left(S_{A} \diamond F(., \varphi(.))(t) \|\right. \\
& \leq\left\|T_{A_{0}}(t) x-x\right\|+V^{\infty}\left(S_{A}, 0, t\right) \sup _{s \in[0, t]}\|F(s, \varphi(s))\| \\
& \leq\left\|T_{A_{0}}(t) x-x\right\|+V^{\infty}\left(S_{A}, 0, \varepsilon\right)\left(\sup _{s \in[0, t]}\|F(s, x)\|+K_{2} \sup _{s \in[0, t]}\|\varphi(s)-x\|\right) \\
& \leq \sup _{t \in[0, \varepsilon]}\left\|T_{A_{0}}(t) x-x\right\|+V^{\infty}\left(S_{A}, 0, \varepsilon\right)\left(\sup _{s \in[0, \varepsilon]}\|F(s, x)\|+K_{2} \varepsilon_{0}\right) .
\end{aligned}
$$

Thus, there exists $\varepsilon_{1} \in\left(0, \varepsilon_{0}\right]$ such that for each $\varepsilon \in\left(0, \varepsilon_{1}\right], \Psi_{1}\left(M_{1}^{\varepsilon}\right) \subset M_{1}^{\varepsilon}$.

Moreover, for each $\varepsilon \in\left(0, \varepsilon_{1}\right]$, each $t \in[0, \varepsilon]$, and each $\left(\varphi_{1}, \varphi_{2}\right) \in M_{1}^{\varepsilon} \times$ $M_{2}^{\varepsilon}$, we have

$$
\begin{aligned}
&\left\|\Psi_{2}\left(\varphi_{1}, \varphi_{2}\right)(t)-\left[A_{0} x+F(0, x)\right]\right\| \\
& \leq\left\|T_{A_{0}}(t)\left[A_{0} x+F(0, x)\right]-\left[A_{0} x+F(0, x)\right]\right\| \\
&+\left\|\left(S_{A} \diamond \partial_{t} F\left(., \varphi_{1}(.)\right)+\partial_{x} F\left(., \varphi_{1}(.)\right) \varphi_{2}(.)\right)(t)\right\| \\
& \leq \sup _{t \in[0, \varepsilon]}\left\|T_{A_{0}}(t)\left[A_{0} x+F(0, x)\right]-\left[A_{0} x+F(0, x)\right]\right\| \\
&+V^{\infty}\left(S_{A}, 0, \varepsilon\right) \sup _{s \in[0, \varepsilon]}\left\|\partial_{t} F\left(s, \varphi_{1}(s)\right)\right\| \\
&+V^{\infty}\left(S_{A}, 0, \varepsilon\right) \sup _{s \in[0, \varepsilon]}\left\|\partial_{x} F\left(s, \varphi_{1}(s)\right)\right\|\left\|\varphi_{2}(.)\right\| \\
& \leq \sup _{t \in[0, \varepsilon]}\left\|T_{A_{0}}(t)\left[A_{0} x+F(0, x)\right]-\left[A_{0} x+F(0, x)\right]\right\| \\
&+V^{\infty}\left(S_{A}, 0, \varepsilon\right)\left\{K_{1}+K_{2}\left[\left\|A_{0} x+F(0, x)\right\|+\varepsilon_{0}\right]\right\} .
\end{aligned}
$$

Therefore, there exists $\varepsilon_{2} \in\left(0, \varepsilon_{1}\right]$ such that for each $\varepsilon \in\left(0, \varepsilon_{2}\right], \Psi_{2}\left(M_{1}^{\varepsilon} \times\right.$ $\left.M_{2}^{\varepsilon}\right) \subset M_{2}^{\varepsilon}$.

Similarly, for each $\varepsilon \in\left(0, \varepsilon_{2}\right], \Psi\left(M_{1}^{\varepsilon} \times M_{2}^{\varepsilon}\right) \subset M_{1}^{\varepsilon} \times M_{2}^{\varepsilon}$. Now we check that $\Psi\left(E^{\varepsilon}\right) \subset E^{\varepsilon}$. Let $\left(\varphi_{1}, \varphi_{2}\right) \in E^{\varepsilon}$. Then $\varphi_{1} \subset C^{1}\left([0, \varepsilon], X_{0}\right)$ and $\varphi_{1}^{\prime}(t)=$ $\varphi_{2}(t)$ for all $t \in[0, \varepsilon]$. Notice that

$$
\Psi_{1}\left(\varphi_{1}\right)(t)=T_{A_{0}}(t) x+\left(S_{A} \diamond F\left(., \varphi_{1}(.)\right)(t) ;\right.
$$

using Lemma 6.2 and the fact that $x \in D\left(A_{0}\right)$ and $F(0, x) \in \overline{D(A)}$, we have

$$
\begin{aligned}
\frac{d \Psi_{1}\left(\varphi_{1}\right)(t)}{d t}= & A_{0} T_{A_{0}}(t) x+T_{A_{0}}(t) F(0, x)+\left(S_{A} \diamond \frac{d}{d t} F\left(., \varphi_{1}(.)\right)(t)\right. \\
= & T_{A_{0}}(t)\left[A_{0} x+F(0, x)\right] \\
& \quad+\left(S_{A} \diamond \partial_{t} F\left(., \varphi_{1}(.)\right)+\partial_{x} F\left(., \varphi_{1}(.)\right) \varphi_{2}(.)\right)(t) .
\end{aligned}
$$


Thus,

$$
\frac{d \Psi_{1}\left(\varphi_{1}\right)(t)}{d t}=\Psi_{2}\left(\varphi_{1}, \varphi_{2}\right)(t)
$$

and $\Psi\left(E^{\varepsilon}\right) \subset E^{\varepsilon}$. Next we apply Lemma 6.3. It remains to verify i) and ii) for some $\varepsilon \in\left(0, \varepsilon_{2}\right]$ small enough. Let $\left(\varphi_{1}, \varphi_{2}\right),\left(\widehat{\varphi}_{1}, \widehat{\varphi}_{2}\right) \in M_{1}^{\varepsilon} \times M_{2}^{\varepsilon}$ be fixed. We have for each $\varepsilon \in\left(0, \varepsilon_{2}\right]$ that

$$
\begin{aligned}
\left\|\Psi_{1}\left(\varphi_{1}\right)(t)-\Psi_{1}\left(\widehat{\varphi}_{1}\right)(t)\right\| & =\left\|\left(S_{A} \diamond F\left(., \varphi_{1}(.)\right)-F\left(., \widehat{\varphi}_{1}(.)\right)\right)(t)\right\| \\
& \leq V^{\infty}\left(S_{A}, 0, \varepsilon\right)\left\|F\left(s, \varphi_{1}(s)\right)-F\left(s, \widehat{\varphi}_{1}(s)\right)\right\| \\
& \leq V^{\infty}\left(S_{A}, 0, \varepsilon\right) K_{2} \sup _{s \in[0, \varepsilon]}\left\|\varphi_{1}(s)-\widehat{\varphi}_{1}(s)\right\| .
\end{aligned}
$$

Thus there exists $\varepsilon_{3} \in\left(0, \varepsilon_{2}\right]$ such that $\delta_{1}:=V^{\infty}\left(S_{A}, 0, \varepsilon_{3}\right) K_{2} \in(0,1)$; we have for each $\varepsilon \in\left(0, \varepsilon_{3}\right]$ that

$$
\left\|\Psi_{1}\left(\varphi_{1}\right)-\Psi_{1}\left(\widehat{\varphi}_{1}\right)\right\|_{\infty,[0, \varepsilon]} \leq \delta_{1}\left\|\varphi_{1}-\widehat{\varphi}_{1}\right\|_{\infty,[0, \varepsilon]} .
$$

Moreover,

$$
\begin{aligned}
& \left\|\Psi_{2}\left(\varphi_{1}, \varphi_{2}\right)(t)-\Psi_{2}\left(\varphi_{1}, \widehat{\varphi}_{2}\right)(t)\right\|=\left\|\left(S_{A} \diamond \partial_{x} F\left(., \varphi_{1}(.)\right)\left(\varphi_{2}(.)-\widehat{\varphi}_{2}\right)\right)(t)\right\| \\
& \leq V^{\infty}\left(S_{A}, 0, \varepsilon\right) K_{2} \sup _{s \in[0, \varepsilon]}\left\|\varphi_{2}(s)-\widehat{\varphi}_{2}(s)\right\| \leq \delta_{1} \sup _{s \in[0, \varepsilon]}\left\|\varphi_{2}(s)-\widehat{\varphi}_{2}(s)\right\|,
\end{aligned}
$$

which implies that

$$
\left\|\Psi_{2}\left(\varphi_{1}, \varphi_{2}\right)(t)-\Psi_{2}\left(\varphi_{1}, \widehat{\varphi}_{2}\right)\right\|_{\infty,[0, \varepsilon]} \leq \delta_{1}\left\|\varphi_{2}-\widehat{\varphi}_{2}\right\|_{\infty,[0, \varepsilon]} .
$$

Hence, for $\varepsilon=\varepsilon_{3}$ we have $\Psi\left(M_{1}^{\varepsilon} \times M_{2}^{\varepsilon}\right) \subset M_{1}^{\varepsilon} \times M_{2}^{\varepsilon}, \Psi\left(E^{\varepsilon}\right) \subset E^{\varepsilon}$ and $\Psi$ satisfies the assumptions of Lemma 6.3. We deduce that there exists $(u, v) \in$ $M_{1}^{\varepsilon} \times M_{2}^{\varepsilon}$ such that for each $\left(\varphi_{1}, \varphi_{2}\right) \in M_{1}^{\varepsilon} \times M_{2}^{\varepsilon}$,

$$
\Psi^{n}\left(\varphi_{1}, \varphi_{2}\right) \rightarrow(u, v) \text { as } n \rightarrow+\infty .
$$

Since $\Psi\left(E^{\varepsilon}\right) \subset E^{\varepsilon}$ and $E^{\varepsilon}$ is closed, we deduce that $(u, v) \in E^{\varepsilon}$. In particular, $u \in C^{1}([0, \varepsilon], X)$, and the result follows.

Lemma 6.5. Let Assumptions 2.1 and 2.8 be satisfied. Let $\tau>0$ be fixed and $F:[0, \tau] \times \overline{D(A)} \rightarrow X$ be continuously differentiable. Assume that there exists an integrated solution $u \in C([0, \tau], X)$ of the Cauchy problem

$$
\frac{d u(t)}{d t}=A u(t)+F(t, u(t)), t \in[0, \tau], u(0)=x \in X_{0} .
$$

Assume in addition that $x \in D\left(A_{0}\right)$ and $F(0, x) \in \overline{D(A)}$. Then

$$
u \in C^{1}([0, \tau], X) \cap C([0, \tau], D(A))
$$


and

$$
u^{\prime}(t)=A u(t)+F(t, u(t)), \quad \forall t \in[0, \tau] .
$$

Proof. Let $w \in C([0, \tau], \overline{D(A)})$ be a solution of the equation

$$
\begin{aligned}
w(t)= & A x+F(0, x)+A \int_{0}^{t} w(s) d s \\
& +\int_{0}^{t} \frac{\partial}{\partial t} F(s, u(s))+D_{x} F(s, u(s)) w(s) d s, \forall t \in[0, \tau] .
\end{aligned}
$$

From the results in Section 4 concerning globally Lipschitz perturbations, it is clear that the solution $w(t)$ exists and is uniquely determined. Since $u(t)$ exists on $[0, \tau]$, let $t \in[0, \tau)$ be fixed. We have for each $h \in(0, \tau-t)$ that

$$
\begin{aligned}
& \frac{u(t+h)-u(t)}{h}=\frac{1}{h} A\left[\int_{0}^{t+h} u(s) d s-\int_{0}^{t} u(s) d s\right] \\
& +\frac{1}{h}\left[\int_{0}^{t+h} F(s, u(s)) d s-\int_{0}^{t} F(s, u(s)) d s\right] \\
& =A\left[\int_{0}^{t} \frac{u(s+h)-u(s)}{h} d s\right]+\frac{1}{h} A \int_{0}^{h} u(s) d s \\
& +\int_{0}^{t} \frac{F(s+h, u(s+h))-F(s, u(s))}{h} d s+\frac{1}{h} \int_{0}^{h} F(s, u(s)) d s .
\end{aligned}
$$

Therefore,

$$
\begin{aligned}
& \frac{u(t+h)-u(t)}{h}-w(t)=A \int_{0}^{t}\left[\frac{u(s+h)-u(s)}{h}-w(s)\right] d s \\
& +\frac{1}{h} A \int_{0}^{h} u(s) d s+\frac{1}{h} \int_{0}^{h} F(s, u(s)) d s-A x-F(0, x) \\
& \quad+\int_{0}^{t}\left[\frac{F(s+h, u(s+h))-F(s+h, u(s))}{h}-D_{x} F(s, u(s)) w(s)\right] d s \\
& +\int_{0}^{t}\left[\frac{F(s+h, u(s))-F(s, u(s))}{h}-\frac{\partial}{\partial t} F(s, u(s))\right] d s .
\end{aligned}
$$

Denote

$$
v_{h}(t):=\frac{u(t+h)-u(t)}{h}-w(t)
$$

and

$$
x_{h}:=\frac{1}{h} A \int_{0}^{h} u(s) d s+\frac{1}{h} \int_{0}^{h} F(s, u(s)) d s-A x-F(0, x) .
$$


We have

$$
\begin{aligned}
v_{h}(t)= & x_{h}+A \int_{0}^{t} v_{h}(s) d s+\int_{0}^{t} \int_{0}^{1} D_{x} F(l(u(s+h)-u(s))+u(s)) \\
& \times\left(\frac{u(s+h)-u(s)}{h}-w(s)\right) d l d s \\
+ & \int_{0}^{t} \int_{0}^{1}\left[D_{x} F(l(u(s+h)-u(s))+u(s))-D_{x} F(u(s))\right] w(s) d l d s \\
+ & \int_{0}^{t}\left[\frac{F(s+h, u(s))-F(s, u(s))}{h}-\frac{\partial}{\partial t} F(s, u(s))\right] d s .
\end{aligned}
$$

Set

$$
K=\sup _{l \in[0,1], s \in[0, \tau], h \in[0, \tau-s]}\left\|D_{x} F(l(u(s+h)-u(s))+u(s))\right\|_{\mathcal{L}\left(X_{0}, X\right)}<+\infty .
$$

Let $\widehat{\tau}>0$ such that

$$
M V^{\infty}\left(S_{A}, 0, t\right) \leq \frac{1}{8(K+1)}, \forall t \in[0, \widehat{\tau}] .
$$

Choose $\gamma>\max \left(0, \omega_{A}\right)$ so that $\frac{1}{4\left(1-e^{\left(\omega_{A}-\gamma\right) \hat{\tau}}\right)}<\frac{1}{2}$. Then by Proposition 2.14, we have for all $\gamma>\max \left(0, \omega_{A}\right)$ that

$$
\begin{aligned}
& e^{-\gamma t}\left\|v_{h}(t)\right\| \leq M\left\|x_{h}\right\|+\frac{1}{2} \sup _{s \in[0, \tau]} e^{-\gamma s}\left\|v_{h}(s)\right\| \\
& \quad+\sup _{s \in[0, \tau]} e^{-\gamma s}\left\|\int_{0}^{1}\left[D_{x} F(l(u(s+h)-u(s))+u(s))-D_{x} F(u(s))\right] w(s) d l\right\| \\
& \quad+\sup _{s \in[0, \tau]} e^{-\gamma s}\left\|\int_{0}^{1}\left[\frac{F(s+h, u(s))-F(s, u(s))}{h}-\frac{\partial}{\partial t} F(s, u(s))\right] d l\right\|,
\end{aligned}
$$

which implies that

$$
\begin{aligned}
& e^{-\gamma t}\left\|v_{h}(t)\right\| \leq 2 M\left\|x_{h}\right\| \\
& +2 \sup _{s \in[0, \tau]} e^{-\gamma s}\left\|\int_{0}^{1}\left[D_{x} F(l(u(s+h)-u(s))+u(s))-D_{x} F(u(s))\right] w(s) d l\right\| \\
& +2 \sup _{s \in[0, \tau]} e^{-\gamma s}\left\|\int_{0}^{1}\left[\frac{F(s+h, u(s))-F(s, u(s))}{h}-\frac{\partial}{\partial t} F(s, u(s))\right] d l\right\| .
\end{aligned}
$$

We now claim that

$$
\lim _{h \searrow 0} x_{h}=0
$$


Indeed, we have

$$
\frac{u(h)-u(0)}{h}=\frac{1}{h} A \int_{0}^{h} u(s) d s+\frac{1}{h} \int_{0}^{h} F(s, u(s)) d s
$$

and by Lemma 6.4, we have

$$
\lim _{h \rightarrow 0^{+}} \frac{u(h)-u(0)}{h}=A x+F(0, x),
$$

so

$$
\lim _{h \searrow 0} x_{h}=0 .
$$

We conclude that for each $t \in[0, \tau)$, we will have

$$
\lim _{h \rightarrow 0^{+}} \frac{u(t+h)-u(t)}{h}=w(t) .
$$

Since $w \in C([0, \tau], X)$, we deduce that $u \in C^{1,+}([0, \tau], X)$. By using Lemma 6.1 , we obtain the result.

To extend the differentiability result to the case where $F\left(0, x_{0}\right) \notin \overline{D(A)}$, we notice that, since $u(t) \in \overline{D(A)}$ for all $t \in[0, T]$, a necessary condition for the differentiability is

$$
A x+F(0, x) \in \overline{D(A)} .
$$

In fact, this condition is also sufficient. Indeed, taking any bounded linear operator $B \in \mathcal{L}(X)$, if $u$ satisfies

$$
u(t)=x+A \int_{0}^{t} u(s) d s+\int_{0}^{t} F(s, u(s)) d s, \forall t \in[0, T]
$$

then we have

$$
u(t)=x+(A+B) \int_{0}^{t} u(s) d s+\int_{0}^{t}(F(s, u(s))-B u(s)) d s, t \in[0, T] .
$$

So to prove the differentiability of $u(t)$ it is sufficient to find $B$ such that $(A+B) x \in \overline{D(A)}$. Take $B(\varphi)=-x^{*}(\varphi) A x$, where $x^{*} \in X^{*}$ is a continuous linear form with $x^{*}(x)=1$ if $x \neq 0$, which is possible by the Hahn-Banach theorem. We then have

$$
x \in D(A)=D(A+B) \text { and }(A+B) x \in \overline{D(A)}=\overline{D(A+B)} .
$$

Moreover, assuming that $A x+F(0, x) \in \overline{D(A)}$, we obtain $F\left(0, x_{0}\right)-B x \in$ $\overline{D(A)}$. By using Theorem 2.12, we deduce that $A+B$ satisfies Assumptions 2.1 and 2.8 and we have the following theorem. 
Theorem 6.6. Let Assumptions 2.1 and 2.8 be satisfied. Let $\tau>0$ be fixed and $F:[0, \tau] \times \overline{D(A)} \rightarrow X$ be continuously differentiable. Assume that there exists an integrated solution $u \in C([0, \tau], X)$ of the Cauchy problem

$$
\frac{d u(t)}{d t}=A u(t)+F(t, u(t)), t \in[0, \tau], u(0)=x \in X_{0} .
$$

Assume in addition that

$$
x \in D(A) \text { and } A x+F(0, x) \in \overline{D(A)} .
$$

Then $u \in C^{1}([0, \tau], X) \cap C([0, \tau], D(A))$ and

$$
u^{\prime}(t)=A u(t)+F(t, u(t)), \quad \forall t \in[0, \tau] .
$$

We now consider the nonlinear generator

$$
A_{N} \varphi=A \varphi+F(0, \varphi), \varphi \in D\left(A_{N}\right)=D(A) .
$$

As in the linear case, one may define $A_{N, 0}$ (the part $A_{N}$ in $\overline{D(A)}$ ) as follows:

$$
A_{N, 0}=A_{N} \text { on } D\left(A_{N, 0}\right)=\left\{y \in D(A): A_{N} y \in \overline{D(A)}\right\} \text {. }
$$

Of course, one may ask about the density of the domain $D\left(A_{N, 0}\right)$ in $\overline{D(A)}$.

Lemma 6.7. Under Assumptions 2.1-2.8 and 3.2, the domain $D\left(A_{N, 0}\right)$ is dense in $X_{0}=\overline{D(A)}$. Assume in addition that $X$ has a positive cone $X_{+}$ and that Assumption 3.4 is satisfied. Then $D\left(A_{N, 0}\right) \cap X_{0+}$ is dense in $X_{0+}$.

Proof. Let $y \in \overline{D(A)}$ be fixed. Consider the following fixed-point problem: $x_{\lambda} \in D(A)$ satisfies

$$
(\lambda I-A-F) x_{\lambda}=\lambda y \Leftrightarrow x_{\lambda}=\lambda(\lambda I-A)^{-1} y+(\lambda I-A)^{-1} F\left(0, x_{\lambda}\right) .
$$

Denote

$$
\Phi_{\lambda}(x)=\lambda(\lambda I-A)^{-1} y+(\lambda I-A)^{-1} F\left(0, x_{\lambda}\right), \quad \forall x \in X_{0} .
$$

Fix $r>0$. Since $y \in \overline{D(A)}$, by Lemma 2.13 , $\lim _{\lambda \rightarrow+\infty}\left\|(\lambda I-A)^{-1}\right\|_{\mathcal{L}(X)}$ $=0$, thus we deduce that there exists $\lambda_{0}>\omega_{A}$ such that

$$
\Phi_{\lambda}\left(B_{X_{0}}(y, r)\right) \subset B_{X_{0}}(y, r), \forall \lambda \geq \lambda_{0},
$$

where $B(y, r)$ denotes the ball centered at $y$ with radius $r$ in $X_{0}$. Moreover, there exists $\lambda_{1} \geq \lambda_{0}$, such that for each $\lambda \geq \lambda_{1}, \Phi_{\lambda}$ is a strict contraction on $B(y, r)$. Hence, for all $\lambda \geq \lambda_{1}$, there exists $x_{\lambda} \in B(y, r)$ such that $\Phi_{\lambda}\left(x_{\lambda}\right)=$ 
$x_{\lambda}$. Finally, using the fact that $y \in \overline{D(A)}$, we have $\lim _{\lambda \rightarrow+\infty} \lambda(\lambda I-A)^{-1} y=y$, SO

$$
\lim _{\lambda \rightarrow+\infty} x_{\lambda}=y .
$$

The proof of the positive case is similar.

\section{Stability of Equilibria}

In this section we first investigate the local stability of an equilibrium.

Proposition 7.1. Let Assumptions 2.1 and 2.8 be satisfied. Let $F: \overline{D(A)} \rightarrow$ $X$ be a continuous map. Assume that

(a) there exists $\bar{x} \in D(A)$ such that $A \bar{x}+F(\bar{x})=0$;

(b) there exist $\widehat{M} \geq 1, \widehat{\omega}<0$, and $L \in \mathcal{L}\left(X_{0}, X\right)$ such that

$$
\left\|T_{(A+L)_{0}}(t)\right\|_{\mathcal{L}\left(X_{0}\right)} \leq \widehat{M} e^{\widehat{\omega} t}, \forall t \geq 0
$$

(c) $\|F-L\|_{\operatorname{Lip}\left(\bar{B}_{X_{0}}(\bar{x}, r), X\right)} \rightarrow 0$ as $r \rightarrow 0$.

Then for each $\gamma \in(\widehat{\omega}, 0)$ there exists $\varepsilon>0$, such that for each $x \in \bar{B}_{X_{0}}(\bar{x}, \varepsilon)$, there exists a unique solution $U(). x \in C\left([0,+\infty), X_{0}\right)$ of

$$
U(t) x=x+A \int_{0}^{t} U(s) x d s+\int_{0}^{t} F(U(s) x) d s, \quad \forall t \geq 0
$$

which satisfies

$$
\|U(t) x-\bar{x}\| \leq e^{\gamma t} 2 \widehat{M}\|x-\bar{x}\|, \quad \forall t \geq 0, \forall x \in X_{0} .
$$

Proof. Without loss of generality we can assume that $\bar{x}=0, L=0, \omega_{A}<0$, and

$$
\|F\|_{\operatorname{Lip}\left(\bar{B}_{X_{0}}(\bar{x} \eta), X\right)} \rightarrow 0 \text { as } \eta \rightarrow 0 .
$$

Choose $\eta_{0}>0$ such that

$$
\|F\|_{\operatorname{Lip}\left(\bar{B}_{X_{0}}\left(\bar{x} \eta_{0}\right), X\right)}<+\infty .
$$

Let $\phi:(-\infty,+\infty) \rightarrow[0,+\infty)$ be a Lipschitz continuous map such that

$$
\phi(\alpha) \begin{cases}=0, & \text { if } 2 \leq|\alpha| \\ \in[0,1], & \text { if } 1 \leq|\alpha| \leq 2 \\ =1, & \text { if }|\alpha| \leq 1 .\end{cases}
$$

Set

$$
F_{r}(x)=\phi(r\|x\|) F(x), \forall x \in X_{0}, \forall r>0 .
$$


Then

$$
F_{r}(x)= \begin{cases}0, & \text { if } \frac{2}{r} \leq\|x\|, \\ F(x), & \text { if }\|x\| \leq \frac{1}{r} .\end{cases}
$$

Choose $\eta \in\left(0, \eta_{0}\right]$ and fix $r=\frac{2}{\eta}$. Let $x, y \in X_{0}$. Define $\varphi:[0,1] \rightarrow \mathbb{R}$ by

$$
\varphi(t)=\left\|F_{r}(t(x-y)+y)-F_{r}(y)\right\|, \forall t \in[0,1] .
$$

Since $\|F\|_{\operatorname{Lip}\left(\bar{B}_{X_{0}}(\bar{x}, \eta), X\right)}<+\infty$, the map $\varphi$ is Lipschitz continuous, thus we have for each pair $t, s \in[0,1]$ that

$$
|\varphi(t)-\varphi(s)| \leq\|F\|_{\operatorname{Lip}\left(\bar{B}_{X_{0}}(\bar{x}, \eta), X\right)}\left(2\|\phi\|_{\operatorname{Lip}}+1\right)\|x-y\||t-s| .
$$

In particular, for $t=1$ and $s=0$, we deduce that

$$
\left\|F_{r}(x)-F_{r}(y)\right\| \leq\|F\|_{\operatorname{Lip}\left(\bar{B}_{X_{0}}\left(\bar{x}, \frac{2}{r}\right), X\right)}\left(2\|\phi\|_{\text {Lip }}+1\right)\|(x-y)\| .
$$

Thus for all $r \geq \frac{2}{\eta_{0}}, F_{r} \in \operatorname{Lip}\left(X_{0}, X\right)$ and

$$
\left\|F_{r}\right\|_{\operatorname{Lip}\left(X_{0}, X\right)} \leq\|F\|_{\operatorname{Lip}\left(\bar{B}_{X_{0}}\left(\bar{x}, \frac{2}{r}\right), X\right)}\left(2\|\phi\|_{\text {Lip }}+1\right) \rightarrow 0 \text { as } r \rightarrow+\infty .
$$

For each $r \geq \frac{2}{\eta_{0}}$, we consider the nonlinear semigroup $\left\{U_{r}(t)\right\}$ which is a solution of

$$
U_{r}(t) x=x+A \int_{0}^{t} U_{r}(s) x d s+\int_{0}^{t} F_{r}\left(U_{r}(s) x\right) d s, \quad \forall t \geq 0 .
$$

Let $\gamma \in(\widehat{\omega}, 0)$ be fixed. By Proposition 4.1 and $(3.5)$, there exists $r_{0}=$ $r_{0}(\gamma) \geq \frac{2}{\eta_{0}}$ such that

$$
\left\|U_{r_{0}}(t) x\right\| \leq e^{\gamma t} 2 M\|x\|, \quad \forall t \geq 0, \forall x \in X_{0} .
$$

Let $\varepsilon \in\left(0, \frac{1}{2 r_{0}} \frac{1}{2 M}\right)$. Then, for each $x \in B_{X_{0}}(0, \varepsilon)$,

$$
\left\|U_{r_{0}}(t) x\right\| \leq e^{\gamma t} 2 M\|x\| \leq \frac{1}{2 r_{0}} .
$$

On the other hand, since $F=F_{r}$ on $B_{X_{0}}\left(0, \frac{1}{2 r_{0}}\right)$, we deduce that for each $x \in B_{X_{0}}(0, \varepsilon), U_{r_{0}}()$.$x is a solution of$

$$
U_{r}(t) x=x+A \int_{0}^{t} U_{r}(s) x d s+\int_{0}^{t} F\left(U_{r}(s) x\right) d s, \quad \forall t \geq 0 .
$$

The uniqueness of the solution with initial value $x$ in $B_{X_{0}}(0, \varepsilon)$ follows from the fact that $F$ is locally Lipschitz continuous around 0 and by using the arguments of Lemma 3.3 in Magal and Ruan [22]. 
Remark 7.2. (1) If $F$ is continuously differentiable in $B_{X_{0}}\left(\bar{x}, r_{0}\right)$, we set $L=D F(\bar{x})$. Then by the formula

$$
F(x)-F(y)=\int_{0}^{1} D F(s(x-y)+y)(x-y) d s, \quad \forall x, y \in B_{X_{0}}(\bar{x}, \varepsilon),
$$

it is clear that

$$
\|F-D F(\bar{x})\|_{\operatorname{Lip}\left(\bar{B}_{X_{0}}(\bar{x}, r), X\right)} \rightarrow 0 \text { as } r \rightarrow 0 .
$$

Thus if $\bar{x}$ is an equilibrium (i.e., assertion (a) is satisfied) and

$$
\left\|T_{(A+D F(\bar{x}))_{0}}(t)\right\|_{\mathcal{L}\left(X_{0}\right)} \leq \widehat{M} e^{\widehat{\omega} t}, \quad \forall t \geq 0
$$

for some $\widehat{M} \geq 1$ and $\widehat{\omega}<0$, the conclusion of the proposition holds.

(2) In order to see an example where the condition (c) is more appropriate than the usual differentiability condition, we consider the following case. Assume that $F$ is quasi-linear; that is, $F(x)=L(x) x$, where $L: X_{0} \rightarrow$ $\mathcal{L}\left(X_{0}, X\right)$ is a Lipschitz continuous map (but not necessarily differentiable in a neighborhood of 0$)$. Then

$$
\begin{aligned}
& \|(F-L(0)) x-(F-L(0)) y\|=\|(L(x)-L(0)) x-(L(y)-L(0)) y\| \\
& \leq\|(L(x)-L(0)) x-(L(y)-L(0)) x\|+\|(L(y)-L(0)) x-(L(y)-L(0)) y\| \\
& \leq[\|x\|+\|y\|]\|L\|_{\text {Lip }}\|x-y\| .
\end{aligned}
$$

So

$$
\|(F-L(0))\|_{\operatorname{Lip}\left(B_{X_{0+}}(0, \varepsilon), X\right)} \leq 2 \varepsilon\|L\|_{\text {Lip }} \rightarrow 0 \text { as } \varepsilon \rightarrow 0 .
$$

Thus, in this case we can apply the condition (c), but $F$ is not differentiable.

We now investigate the global asymptotic stability of an equilibrium.

Proposition 7.3. Let Assumptions 2.1 and 2.8 be satisfied. Let $F: \overline{D(A)} \rightarrow$ $X$ be a Lipschitz continuous map. Assume that

(a) there exists $\bar{x} \in D(A)$ such that $A \bar{x}+F(\bar{x})=0$;

(b) there exist $\widehat{M}>0, \widehat{\omega}<0$, and $L \in \mathcal{L}\left(X_{0}, X\right)$, such that

$$
\left\|T_{(A+L)_{0}}(t)\right\|_{\mathcal{L}\left(X_{0}\right)} \leq \widehat{M} e^{\widehat{\omega} t}, \forall t \geq 0
$$

Consider $\{U(t)\}_{t \geq 0}$ the $C_{0}$-semigroup of nonlinear operators on $X_{0}$ which is the solution of

$$
U(t) x=x+A \int_{0}^{t} U(s) x d s+\int_{0}^{t} F(U(s) x) d s, \quad \forall t \geq 0 .
$$


Then, for each $\gamma \in(\widehat{\omega}, 0)$, there exists $\delta_{0}=\delta_{0}(\gamma)>0$, such that

$$
\|F-L\|_{\operatorname{Lip}\left(X_{0}, X\right)} \leq \delta_{0} \Rightarrow\|U(t) x-\bar{x}\| \leq e^{\gamma t} 2 \widehat{M}\|x-\bar{x}\|, \forall t \geq 0, \forall x \in X_{0} .
$$

Thus $\bar{x}$ is a globally exponentially stable equilibrium of $\{U(t)\}_{t \geq 0}$.

Proof. Replacing $U(t) x$ by $V(t) x=U(t)(x+\bar{x})-\bar{x}$ and $F($.$) by G()=$. $F(.+\bar{x})-F(\bar{x})$, respectively, without loss of generality we can assume that $\bar{x}=0$. Moreover, using Theorem 2.12 and replacing $M$ by $\widehat{M}, \omega_{A}$ by $\widehat{\omega}, A$ by $A+L$, and $F$ by $F-L$, respectively, we can further assume that $L=0$ and $\omega_{A}<0$.

Fix $\tau>0$ and set $\varepsilon:=M \delta(\tau)$. Let $\gamma \in\left(\omega_{A}, 0\right)$ be fixed. Choose $\delta_{0}=$ $\delta_{0}(\gamma)>0$ such that

$$
\delta_{0} \frac{2 \varepsilon e^{-\gamma \tau_{\varepsilon}}}{\left(1-e^{\left.\left(\omega_{A}-\gamma\right) \tau_{\varepsilon}\right)}\right.} \leq \frac{1}{2}
$$

Then by Lemma 2.15 we have

$$
\left\|\mathcal{L}_{0}(\varphi)\right\|_{\mathcal{L}\left(B C^{\gamma}([0,+\infty), X), B C^{\gamma}\left([0,+\infty), X_{0}\right)\right)} \leq \frac{2 \varepsilon e^{-\gamma \tau_{\varepsilon}}}{\left(1-e^{\left(\omega_{A}-\gamma\right) \tau_{\varepsilon}}\right)} \leq \frac{1}{2 \delta_{0}} .
$$

It is sufficient to consider the problem $U(). x \in B C^{\gamma}\left([0,+\infty), X_{0}\right)$,

$$
U(t) x=T_{A_{0}}(t) x+\mathcal{L}_{0}(\Psi(U(.) x))(t), \forall t \in[0,+\infty),
$$

where $\Psi: B C^{\gamma}\left([0,+\infty), X_{0}\right) \rightarrow B C^{\gamma}([0,+\infty), X)$ is defined by

$$
\Psi(\varphi)(t)=F(\varphi(t)), \quad \forall t \in[0,+\infty) .
$$

If $\|F\|_{\operatorname{Lip}\left(X_{0}, X\right)} \leq \delta_{0}$, we have $\left\|\mathcal{L}_{0} \circ \Psi\right\|_{\operatorname{Lip}\left(B C^{\gamma}\left([0,+\infty), X_{0}\right), B C^{\gamma}\left([0,+\infty), X_{0}\right)\right)} \leq$ $1 / 2$, so for each $t \geq 0$

$$
\|U(.) x\|_{B C^{\gamma}\left([0,+\infty), X_{0}\right)} \leq M\|x\|+\frac{1}{2}\|U(.) x\|_{B C^{\gamma}\left([0,+\infty), X_{0}\right)}
$$

and the result follows.

Let $L: X_{0} \rightarrow X$ be a bounded linear operator. In order to apply the stability theorem, one needs to prove that there exist two constants $\widehat{M} \geq 1$ and $\widehat{\omega}<0$, such that

$$
\left\|T_{(A+L)_{0}}(t)\right\|_{\mathcal{L}\left(X_{0}\right)} \leq \widehat{M} e^{\widehat{\omega} t}, \quad \forall t \geq 0,
$$

which is also equivalent to

$$
\omega_{0}\left((A+L)_{0}\right):=\lim _{t \rightarrow+\infty} \frac{\ln \left(\left\|T_{(A+L)_{0}}(t)\right\|_{\mathcal{L}\left(X_{0}\right)}\right)}{t}<\widehat{\omega}
$$


This condition can also be expressed by using spectral properties of the linear operator $A+L$. In fact, by applying the results obtained by Webb [43] (see also Engel and Nagel [14] for more results), it is necessary to verify the following two properties:

(a) (Point spectrum condition)

$$
\sigma_{p}(A+L) \subset\{\lambda \in \mathbb{C}: \operatorname{Re}(\lambda)<\widehat{\omega}\}
$$

where

$$
\sigma_{p}(A+L)=\{\lambda \in \mathbb{C}: N(\lambda I-(A+L)) \neq\{0\}\} .
$$

(b) (Essential growth rate condition)

$$
\omega_{0, \mathrm{ess}}\left((A+L)_{0}\right):=\lim _{t \rightarrow+\infty} \frac{\ln \left(\left\|T_{(A+L)_{0}}(t)\right\|_{\mathrm{ess}}\right)}{t}<\widehat{\omega},
$$

where

$$
\left\|T_{(A+L)_{0}}(t)\right\|_{\text {ess }}=\kappa\left(T_{(A+L)_{0}}(t) B_{X_{0}}(0,1)\right)
$$

with $B_{X_{0}}(0,1)=\left\{x \in X_{0}:\|x\| \leq 1\right\}$, and for each bounded set $B \subset$ $X, \kappa(B)=\inf \{\varepsilon>0: B$ can be covered by a finite number of balls of radius $\leq \varepsilon\}$ is the Kuratovsky measure of non-compactness.

In practice the essential growth rate condition can be studied by using perturbation techniques. When $R(L) \subset X_{0}$ this question has been investigated by Webb $[41,42,43]$. When $R(L) \nsubseteq X_{0}$, this question has been investigated in the Hille-Yosida case by Thieme [37] and extended to the non-Hille-Yosida case by Ducrot et al. [12].

As a consequence of Theorem 2.2 in Desch and Schappacher [9] and Proposition 5.1, we have the following result on the instability of an equilibrium (see Thieme [35, Corollary 4.3]).

Proposition 7.4. Let Assumptions 2.1 and 2.8 be satisfied. Let $F: \overline{D(A)} \rightarrow$ $X$ be a Lipschitz continuous map. Assume that there exists $\bar{x} \in D(A)$ such that $A \bar{x}+F(\bar{x})=0$. Assume that $\omega_{0, \text { ess }}\left((A+D F(\bar{x}))_{0}\right)<0$ and there exists $\lambda \in \sigma_{p}\left((A+D F(\bar{x}))_{0}\right)$ with $\operatorname{Re}(\lambda)>0$. Then $\bar{x}$ is an unstable equilibrium in the following sense: There exist a constant $\varepsilon>0$ and a sequence $x_{n}\left(\in X_{0}\right) \rightarrow \bar{x}$ as $t_{n} \rightarrow+\infty$, such that

$$
\left\|U\left(t_{n}\right) x_{n}-\bar{x}\right\| \geq \varepsilon \text { for all } n \geq 0 .
$$

Remark 7.5. When the property of an equilibrium changes from stability to instability, interesting and complex dynamics, such as Hopf bifurcation, 
can occur. We refer to Magal and Ruan [23] for detailed results on this aspect.

\section{Applications}

As we mentioned in the Introduction, the key point in applying our results is to verify the main Assumptions 2.1 and 2.8. In this section, as examples we consider transport equations and parabolic equations and verify that the Assumptions 2.1 and 2.8 hold, so that the theory developed in this article applies.

8.1. Transport equations. First consider the transport equation with a nonlinear (and nonlocal) boundary condition

$$
\left\{\begin{array}{l}
\frac{\partial u}{\partial t}+\frac{\partial u}{\partial x}=M(u(t, .))(x), \quad t>0, a>0 \\
u(t, 0)=G(u(t, .)) \\
u(0, .)=u_{0} \in L^{p}((0,+\infty), \mathbb{R}),
\end{array}\right.
$$

where $M: L^{p}((0,+\infty), \mathbb{R}) \rightarrow L^{p}((0,+\infty), \mathbb{R})$ and $G: L^{p}((0,+\infty), \mathbb{R}) \rightarrow \mathbb{R}$ are continuous maps.

In order to take into account the nonlinear boundary condition we consider the extended state space $X=\mathbb{R} \times L^{p}((0,+\infty), \mathbb{R})$ endowed with the usual product norm and a linear operator $A: D(A) \subset X \rightarrow X$ defined by

$$
A\left(\begin{array}{l}
0 \\
\varphi
\end{array}\right)=\left(\begin{array}{c}
-\varphi(0) \\
-\varphi^{\prime}
\end{array}\right)
$$

with $D(A)=\left\{0_{\mathbb{R}}\right\} \times W^{1, p}((0,+\infty), \mathbb{R})$. Notice that $X_{0}=\overline{D(A)} \neq X$. So $A$ is non-densely defined in $X$. Also, note that $A$ is Hille-Yosida if and only if $p=1$. So $A$ is not a Hille-Yosida operator if we assume $p>1$.

Now we consider a nonlinear operator $F: \overline{D(A)} \rightarrow X$ defined by

$$
F\left(\left(\begin{array}{c}
0 \\
\varphi
\end{array}\right)\right)=\left(\begin{array}{c}
G(\varphi) \\
M(\varphi)
\end{array}\right)
$$

Then by identifying $u(t,$.$) to v(t)=\left(\begin{array}{c}0 \\ u(t, .)\end{array}\right)$, the PDE (8.1) can be formulated as a non-densely defined Cauchy problem

$$
\frac{d v(t)}{d t}=A v(t)+F(v(t)) \text { for } t \geq 0 \text { with } u(0)=x \in \overline{D(A)} .
$$

Lemma 8.1. The operator A satisfies Assumption 2.1. 
Proof. One may readily check that the resolvent is given by the formula

$$
(\lambda I-A)^{-1}\left(\begin{array}{c}
y \\
\psi
\end{array}\right)=\left(\begin{array}{c}
0 \\
\varphi
\end{array}\right) \Leftrightarrow \varphi(a)=e^{-\lambda a} y+\int_{0}^{a} e^{-\lambda(a-s)} \psi(s) d s .
$$

It follows that

$$
\left\|(\lambda I-A)^{-1}\left(\begin{array}{l}
y \\
0
\end{array}\right)\right\|=\left(\frac{1}{p \lambda}\right)^{1 / p}|y| .
$$

By using Young's inequality we also have

$$
\left\|(\lambda I-A)^{-1}\left(\begin{array}{c}
0 \\
\psi
\end{array}\right)\right\|=\frac{1}{\lambda}\|\psi\|_{L^{p}} .
$$

So $A_{0}$, the part of $A$ in $X_{0}=\overline{D(A)}$, is a Hille-Yosida operator and we have the estimate

$$
0<\lim _{\lambda \rightarrow+\infty} \lambda^{1 / p}\left\|(\lambda I-A)^{-1}\right\|_{\mathcal{L}(X)}<+\infty .
$$

This completes the proof.

Now we can claim that $A_{0}$ generates a $C_{0}$-semigroup $\left\{T_{A_{0}}(t)\right\}_{t \geq 0}$ and $A$ generates an integrated semigroup $S_{A}(t)$.

Lemma 8.2. $\left\{T_{A_{0}}(t)\right\}_{t>0}$, the $C_{0}$-semigroup generated by $A_{0}$ (the part of $A$ in $\left.X_{0}\right)$, is defined by

$$
T_{A_{0}}(t)\left(\begin{array}{c}
0 \\
\varphi
\end{array}\right)=\left(\begin{array}{c}
0 \\
\widehat{T}_{A_{0}}(t) \varphi
\end{array}\right)
$$

with

$$
\widehat{T}_{A_{0}}(t)(\varphi)(a)= \begin{cases}e^{-\lambda a} \varphi(a-t) & \text { if } a \geq t, \\ 0 & \text { if } a<t .\end{cases}
$$

Moreover, the integrated semigroup $\left\{S_{A}(t)\right\}_{t \geq 0}$ generated by $A$ is defined by

$$
S_{A}(t)\left(\begin{array}{c}
y \\
\varphi
\end{array}\right)=\left(\begin{array}{c}
0 \\
W(t) y+\int_{0}^{t} \widehat{T}_{B_{0}}(s) \varphi d s
\end{array}\right)
$$

with

$$
W(t)(y)(a)= \begin{cases}e^{-\lambda a} y & \text { if } a \leq t \\ 0 & \text { if } a>t .\end{cases}
$$

Proof. For $T_{A_{0}}(t)$ and $S_{A}(t)$ defined by the above formulas, we have

$$
\frac{d}{d t}(\lambda I-A)^{-1} T_{A_{0}}(t) x=\lambda(\lambda I-A)^{-1} T_{A_{0}}(t) x-T_{A_{0}}(t) x
$$


and

$$
\frac{d}{d t}(\lambda I-A)^{-1} S_{A}(t) x=\lambda(\lambda I-A)^{-1} S_{A}(t) x-S_{A}(t) x+(\lambda I-A)^{-1} x .
$$

The result follows from Lemma 2.10 of Magal and Ruan [22].

Lemma 8.3. For each $\tau$, each $f \in L^{p}\left((0, \tau), X_{0}\right)$, and each $x \in X_{0}$, there exists a unique integrated solution $u \in C\left([0, \tau], X_{0}\right)$ of the Cauchy problem

$$
\frac{d u}{d t}=A u(t)+f(t), \quad t \in[0, \tau], u(0)=x,
$$

given by

$$
u(t)=T_{A_{0}}(t) x+\left(S_{A} \diamond f\right)(t), \quad \forall t \in[0, \tau] .
$$

Moreover, there is a constant $M_{\tau}$ such that

$$
\left\|\left(S_{A} \diamond f\right)(t)\right\| \leq M_{\tau}\left(\int_{0}^{t}\|f(s)\|^{p} d s\right)^{1 / p}, \forall t \in[0, \tau] .
$$

Proof. Let $\psi \in C_{c}^{\infty}\left((0,+\infty), Y^{*}\right)$ be fixed. We define $x^{*} \in X_{0}^{*}$ by

$$
x^{*}\left(\begin{array}{c}
0 \\
\varphi
\end{array}\right)=\int_{0}^{+\infty} \psi(s)(\varphi(s)) d s .
$$

Let $x=\left(\begin{array}{c}y \\ \varphi\end{array}\right) \in X$. For each $\lambda>\omega$, we have

$$
x^{*}\left((\lambda I-A)^{-1}\left(\begin{array}{c}
y \\
\varphi
\end{array}\right)\right)=\int_{0}^{+\infty} e^{(-\lambda+\omega) t} W_{x^{*}}(t)(y) d t
$$

with

$$
W_{x^{*}}(t)(y)=e^{-(\lambda+\omega) t} \psi(t) y
$$

Therefore,

$$
\begin{aligned}
x^{*}\left((\lambda I-A)^{-n}\left(\begin{array}{c}
y \\
\varphi
\end{array}\right)\right) & =\frac{(-1)^{n-1}}{(n-1) !} \frac{d^{n-1}}{d \lambda^{n-1}} x^{*}\left((\lambda I-A)^{-1}\left(\begin{array}{c}
y \\
\varphi
\end{array}\right)\right) \\
& =\frac{1}{(n-1) !} \int_{0}^{+\infty} t^{n-1} e^{(-\lambda+\omega) t} W_{x^{*}}(t)(y) d t
\end{aligned}
$$

and

$$
\left|x^{*}\left((\lambda I-A)^{-n}\left(\begin{array}{c}
y \\
\varphi
\end{array}\right)\right)\right| \leq \frac{1}{(n-1) !} \int_{0}^{+\infty} t^{n-1} e^{-\lambda t} M\|\psi(t)\|_{Y^{*}} d t\|y\|_{Y} .
$$

Hence, Proposition 4.1 and Theorem 4.7 in Magal and Ruan [22] imply that $u(t)$ defined by (8.4) is an integrated solution of (8.3). Moreover, by using Lemma 2.10 in Magal and Ruan [22], we obtain the inequality in (8.5). 
Now, by combining Theorem 2.9 and Lemma 8.3, we know that Assumption 2.8 is satisfied. Thus, the theory developed in sections 2-7 applies to the transport equation (8.1).

8.2. Parabolic equations. In parabolic equations, due to the boundary conditions it is well known that elliptic operators are not in general HilleYosida operators but almost sectorial operators (see Da Prato [6], Okazawa [29], Periago and Straub [31] and references therein). To illustrate this property we consider the parabolic system

$$
\left\{\begin{array}{l}
\frac{\partial u}{\partial t}=\frac{\partial^{2} u}{\partial x^{2}}+M(u(t, .))(x), t>0, x>0 \\
-\frac{\partial u(t, 0)}{\partial x}=G(u(t, .)) \\
u(0, .)=u_{0} \in L^{p}((0,+\infty), \mathbb{R}) .
\end{array}\right.
$$

As before we consider a linear operator $A: D(A) \subset X \rightarrow X$ defined by

$$
A\left(\begin{array}{c}
0 \\
\varphi
\end{array}\right)=\left(\begin{array}{c}
\varphi^{\prime}(0) \\
\varphi^{\prime \prime}
\end{array}\right)
$$

with $D(A)=\left\{0_{\mathbb{R}}\right\} \times W^{2, p}((0,+\infty), \mathbb{R})$. One may observe that $A_{0}$, the part of $A$ in $\overline{D(A)}=\left\{0_{\mathbb{R}}\right\} \times L^{p}((0,+\infty), \mathbb{R})$, is the generator of the strongly continuous semigroup of bounded linear operators associated to

$$
\left\{\begin{array}{l}
\frac{\partial u}{\partial t}=\frac{\partial^{2} u}{\partial x^{2}}, \quad t>0, \quad x>0 \\
-\frac{\partial u(t, 0)}{\partial x}=0 \\
u(0, .)=u_{0} \in L^{p}((0,+\infty), \mathbb{R}) .
\end{array}\right.
$$

That is,

$$
A_{0}\left(\begin{array}{c}
0 \\
\varphi
\end{array}\right)=\left(\begin{array}{c}
0 \\
\varphi^{\prime \prime}
\end{array}\right)
$$

with

$$
D\left(A_{0}\right)=\left\{\left(\begin{array}{c}
0 \\
\varphi
\end{array}\right) \in\left\{0_{\mathbb{R}}\right\} \times W^{2, p}((0,+\infty), \mathbb{R}): \varphi^{\prime}(0)=0\right\} .
$$

In particular it is well known that $A_{0}$ is the infinitesimal generator of an analytic semigroup on $\overline{D(A)}$. The resolvent of $A$ is defined by the formula

$$
(\lambda I-A)^{-1}\left(\begin{array}{c}
\alpha \\
\psi
\end{array}\right)=\left(\begin{array}{l}
0 \\
\varphi
\end{array}\right)
$$




$$
\begin{aligned}
\Leftrightarrow \varphi(x) & =\frac{\alpha}{-\sqrt{\lambda}} e^{-\sqrt{\lambda} x} \alpha \\
& +\frac{1}{2 \sqrt{\lambda}} \int_{0}^{+\infty} e^{-\sqrt{\lambda} s} \psi(s) d s e^{-\sqrt{\lambda} x}+\frac{1}{2 \sqrt{\lambda}} \int_{0}^{+\infty} e^{-\sqrt{\lambda}|x-s|} \psi(s) d s
\end{aligned}
$$

for $\lambda \in \mathbb{C}$ with $\operatorname{Re}(\lambda)>0$.

So as in the hyperbolic case we obtain

$$
\begin{aligned}
0 & <\liminf _{\lambda \rightarrow+\infty} \lambda^{1 / p^{*}}\left\|(\lambda I-A)^{-1}\right\|_{\mathcal{L}(X)} \\
& <\limsup _{\lambda \rightarrow+\infty} \lambda^{1 / p^{*}}\left\|(\lambda I-A)^{-1}\right\|_{\mathcal{L}(X)}<+\infty
\end{aligned}
$$

where $p^{*}=\frac{2 p}{1+p}$. Note that $A$ is non-densely defined and is not a Hille-Yosida operator when $p \in(1,+\infty)$.

Now by identifying $u(t,$.$) to v(t)=\left(\begin{array}{c}0 \\ u(t, .)\end{array}\right)$, the PDE (8.6) can be formulated as a non-densely defined Cauchy problem

$$
\frac{d v(t)}{d t}=A v(t)+F(v(t)) \text { for } t \geq 0 \text { with } u(0)=x \in \overline{D(A)} .
$$

We make the following assumption.

Assumption 8.4. Let $A: D(A) \subset X \rightarrow X$ be a linear operator on a Banach space $X$. Assume that there exist two constants, $\omega_{A} \in \mathbb{R}$ and $M_{A}>$ 0 , such that

(a) $\rho\left(A_{0}\right) \supset\left\{\lambda \in \mathbb{C}: \operatorname{Re}(\lambda)>\omega_{A}\right\}$ and

$$
\left\|\left(\lambda-\omega_{A}\right)\left(\lambda I-A_{0}\right)^{-1}\right\|_{\mathcal{L}\left(X_{0}\right)} \leq M_{A}, \forall \lambda \in \mathbb{C}, \operatorname{Re}(\lambda)>\omega_{A}
$$

(b) $\left(\omega_{A},+\infty\right) \subset \rho(A)$ and there exists $p^{*} \geq 1$ such that

$$
\limsup _{\lambda \rightarrow+\infty} \lambda^{1 / p^{*}}\left\|(\lambda I-A)^{-1}\right\|_{\mathcal{L}(X)}<+\infty
$$

The above assumption can be reformulated by saying that $A_{0}$ is sectorial and $A$ is $\frac{1}{p^{*}}$-almost sectorial. Moreover, we have the following result (see Ducrot, Magal and Prevost [13, Theorem 3.1]).

Lemma 8.5. Let Assumption 8.4 be satisfied. Let $\lambda>\omega_{A}$ and $\hat{p} \in\left(p^{*},+\infty\right)$ be fixed. Then, for each $f \in L^{\hat{p}}((0, \tau), X)$, the map $t \rightarrow\left(S_{A} * f\right)(t)$ is continuously differentiable, $\left(S_{A} * f\right)(t) \in D(A)$ for all $t \in[0, \tau]$, and if we 
denote by $u(t)=\frac{d}{d t}\left(S_{A} * f\right)(t)$, then

$$
u(t)=A \int_{0}^{t} u(s) d s+\int_{0}^{t} f(s) d s, \forall t \in[0, \tau] .
$$

Moreover, for each $\beta \in\left(1-\frac{1}{p^{*}}, 1-\frac{1}{\hat{p}}\right)$ and each $t \in[0, \tau]$, the following estimate holds:

$$
\left\|\left(S_{A} \diamond f\right)(t)\right\| \leq M_{\beta, \tau} \int_{0}^{t}(t-s)^{-\beta} e^{\omega_{A}(t-s)}\|f(s)\| d s,
$$

where $M_{\beta, \tau}$ is some positive constant.

Lemma 8.5 implies that, for each $\widehat{p}>p^{*}$ and each $\tau>0$, the Cauchy problem (8.7) has a unique integrated solution and there exists a constant $M_{\tau, \widehat{p}}>0$ such that

$$
\left\|\left(S_{A} \diamond f\right)(t)\right\| \leq M_{\tau, \widehat{p}}\left(\int_{0}^{t}\|f(s)\|^{\widehat{p}} d s\right)^{1 / \widehat{p}}, \quad \forall t \in[0, \tau]
$$

Therefore, Assumptions 2.1 and 2.8 are satisfied for the parabolic equation (8.8). Thus, the results in sections 3-7 apply to this equation too.

For parabolic problems in dimension $n$, we refer to Tanabe [34, Section 3.8, p.82], Agranovich [1], and Volpert and Volpert [40] for general estimates for the resolvent of elliptic operators in the $n$-dimensional case.

The goal of this paper was to develop a comprehensive semilinear theory for non-densely defined Cauchy problems when the linear operator is not a Hille-Yosida operator. The above two examples on transport equations with nonlinear boundary conditions and parabolic equations with nonlocal boundary conditions demonstrate that when the linear operator is not Hille-Yosida, we still can formulate the problems into a non-densely defined Cauchy problem and apply the theory of this paper to study these two types of equations. We believe that our results can be applied to discuss various other types of equations, such as retarded and neutral functional differential equations (see Liu, Magal and Ruan [19], Magal and Ruan [22], and Ducrot, Liu and Magal [11]).

Acknowledgments. We are very grateful to Horst Thieme for his valuable comments and helpful suggestions which helped us to improve the presentation of the paper. We also would like to thank the referee for his helpful comments. 


\section{REFERENCES}

[1] M. S. Agranovich, Elliptic boundary value problems, in "Partial Differential Equations, IX," Encyclopaedia Math. Sci. Vol. 79, Springer, Berlin, 1997, 1-144.

[2] W. Arendt, Resolvent positive operators, Proc. London Math. Soc., 54 (1987), 321349.

[3] W. Arendt, Vector valued Laplace transforms and Cauchy problems, Israel J. Math. 59 (1987), 327-352.

[4] W. Arendt, C. J. K. Batty, M. Hieber, and F. Neubrander, "Vector-Valued Laplace Transforms and Cauchy Problems," Birkhäuser, Basel, 2001.

[5] T. Cazenave and A. Haraux, "An Introduction to Semilinear Evolution Equations," Oxford Lect. Ser. Math. Appl. 13, Oxford, 1998.

[6] G. Da Prato, Semigruppi di crescenca n, Ann. Scuola Norm. Sup. Pisa (3) 20 (1966), 753-782.

[7] G. Da Prato and E. Sinestrari, Differential operators with non-dense domain, Ann. Sc. Norm. Super. Pisa Cl. Sci. 14 (1987), 285-344.

[8] E. B. Davies and M. M. H. Pang, The Cauchy problem and a generalization of the Hille-Yosida theorem, Proc. London Math. Soc. 55 (1987), 181-208.

[9] W. Desch and W. Schappacher, Linearized stability for non linear semigroups, in "Differential Equations in Banach Spaces," ed. by A. Favini and E. Obrecht, Lect. Note Math. 1223, Springer, Berlin, 1986, 61-73.

[10] W. Desch and W. Schappacher, Some generation results for perturbed semigroups, in "Semigroup Theory and Applications," ed. by P. Clément, S. Invernizzi, E. Mitidieri, and I. I. Vrabie, Lect. Notes Pure Appl. Math. 116, Dekker, New York, 1989, 125-152.

[11] A. Ducrot, Z. Liu, and P. Magal, Projectors on the generalized eigenspaces for neutral functional differential equations in $L^{p}$ spaces, Can. J. Math. (to appear).

[12] A. Ducrot, Z. Liu, and P. Magal, Essential growth rate for bounded linear perturbation of non-densely defined Cauchy problems, J. Math. Anal. Appl. 341 (2008), 501-518.

[13] A. Ducrot, P. Magal, and K. Prevost, Integrated semigroups and parabolic equations. Part I: Linear perturbation of almost sectorial operators, submitted (2009).

[14] K.-J. Engel and R. Nagel, "One Parameter Semigroups for Linear Evolution Equations," Springer-Verlag, New York, 2000.

[15] M. Hieber, Integrated semigroups and differential operators on $L^{p}$ spaces, Math. Ann. 291 (1991), 1-16.

[16] M. Hieber, Integrated semigroups and the Cauchy problem for systems in $L^{p}$ spaces, J. Math. Anal. Appl. 162 (1991), 300-308.

[17] M. W. Hirsch and H. Smith, Monotone dynamical systems, Handbook of Differential Equations: Ordinary Differential Equations, Vol. 2, ed. by A. Canada, P. Drabek and A. Fonda, Elsevier, 2005, 239-357.

[18] H. Kellermann and M. Hieber, Integrated semigroups, J. Funct. Anal. 84 (1989), $160-180$.

[19] Z. Liu, P. Magal, and S. Ruan, Projectors on the generalized eigenspaces for functional differential equations using integrated semigroups, J. Differential Equations 244 (2008), 1784-1809.

[20] A. Lunardi, "Analytic Semigroup and Optimal Regularity in Parabolic Problems," Birkhauser, Basel, 1995. 
[21] P. Magal, Compact attractors for time periodic age-structured population models, Electr. J. Differential Equations 2001 (2001), No. 65, 1-35.

[22] P. Magal and S. Ruan, On integrated semigroups and age structured models in $L^{p}$ spaces, Differential Integral Equations 20 (2007), 197-139.

[23] P. Magal and S. Ruan, "Center Manifolds for Semilinear Equations with Non-dense Domain and Applications on Hopf Bifurcation in Age Structured Models," Mem. Amer. Math. Soc. (in press).

[24] P. Magal and H. R. Thieme, Eventual compactness for a semiflow generated by an age-structured models, Comm. Pure Appl. Anal. 3 (2004), 695-727.

[25] R. H. Martin, "Nonlinear Operators and Differential Equations in Banach Spaces," John Wiley and Sons, New York, 1977.

[26] R. H. Martin and H. L. Smith, Abstract functional differential equations and reactiondiffusion systems, Trans. Amer. Math. Soc. 321 (1990), 1-44.

[27] R. H. Martin and H. L. Smith, Reaction-diffusion systems with time delays: Monotonicity, invariance, comparison and convergence, J. Reine Angew. Math. 413 (1991), $1-35$.

[28] F. Neubrander, Integrated semigroups and their application to the abstract Cauchy problem, Pac. J. Math. 135 (1988), 111-155.

[29] N. Okazawa, A generation theorem for semigroups of growth order, Tôhoku Math. J. 26 (1974), 39-51.

[30] A. Pazy, "Semigroups of Linear Operator and Applications to Partial Differential Equations," Springer-Verlag, New York, 1983.

[31] F. Periago and B. Straub, A functional calculus for almost sectorial operators and applications to abstract evolution equations, J. Evol. Equ. 2 (2002), 41-68.

[32] K. Prevost, Modèles épidémiologiques de type paraboliques : Application à l'étude de la propagation de Salmonelles en élevage industriel, $\mathrm{PhD}$ thesis, University of Le Havre, 2008.

[33] I. E. Segal, Nonlinear semigroups, Ann. Math. 78 (1963), 339-364.

[34] H. Tanabe, "Equations of Evolution," Monographs and Studies in Mathematics, no. 6, Pitman, London, 1979.

[35] H. R. Thieme, Semiflows generated by Lipschitz perturbations of non-densely defined operators, Differential Integral Equations 3 (1990), 1035-1066.

[36] H. R. Thieme, Integrated semigroups and integrated solutions to abstract Cauchy problems, J. Math. Anal. Appl. 152 (1990), 416-447.

[37] H. R. Thieme, Quasi-compact semigroups via bounded perturbation, in "Advances in Mathematical Population Dynamics-Molecules, Cells and Man," ed. by O. Arino, D. Axelrod and M. Kimmel, Ser. Math. Biol. Med., 6, World Sci. Publ., River Edge, NJ, 1997, 691-711.

[38] H. R. Thieme, Differentiability of convolutions, integrated semigroups of bounded semivariation, and the inhomogeneous Cauchy problem, J. Evol. Equ. 8 (2008), 283-305.

[39] A. Vanderbauwhede, Center manifold, normal forms and elementary bifurcations, Dynamics Reported - New Series 2 (1989), 89-169.

[40] A. Volpert and V. Volpert, Elliptic problems with a parameter in unbounded domains, Adv. Differential Equations 12 (2007), 573-600.

[41] G. F. Webb, Compactness of bounded trajectories of dynamical systems in infinite dimensional spaces, Proc. Roy. Soc. Edinburgh. 84A (1979), 19-33. 
[42] G. F. Webb, "Theory of Nonlinear Age-dependent Population Dynamics," Marcel Dekker, New York, 1985.

[43] G. F. Webb, An operator-theoretic formulation of asynchronous exponential growth, Trans. Amer. Math. Soc. 303 (1987), 155-164.

[44] F. B. Weissler, Semilinear evolution equations in Banach spaces, J. Funct. Anal., 32 (1979), 277-296.

[45] T.-J. Xiao and J. Liang, "The Cauchy Problem for Higher Order Abstract Differential Equations," Lect. Notes Math. 1701, Springer-Verlag, Berlin, 1998. 